\title{
Driving Rho GTPase activity in endothelial cells regulates barrier integrity
}

\author{
Cora M. L. Beckers; Victor W. M. van Hinsbergh; Geerten P. van Nieuw Amerongen \\ Department for Physiology, VU University Medical Center, Institute for Cardiovascular Research, Amsterdam, the Netherlands
}

\begin{abstract}
Summary
In the past decade understanding of the role of the Rho GTPases RhoA, Rac1 and Cdc42 has been developed from regulatory proteins that regulate specific actin cytoskeletal structures - stress fibers, lamellipodia and filopodia - to complex integrators of cytoskeletal structures that can exert multiple functions depending on the cellular context. Fundamental to these functions are three-dimensional complexes between the individual Rho GTPases, their specific activators (GEFs) and inhibitors (GDIs and GAPs), which greatly outnumber the Rho GTPases themselves, and additional regulatory proteins. By this complexity of regulation different vasoactive mediators can induce various cytoskeletal structures that enable the endothelial cell (EC) to respond adequately. In this review we have focused on this complexity and the consequences of Rho GTPase regulation for endothelial barrier function. The permeability inducers thrombin and VEGF are presented as examples of G-protein coupled receptor- and tyrosine kinase receptor-
\end{abstract}

mediated Rho GTPase activation, respectively. These mediators induce complex but markedly different networks of activators, inhibitors and effectors of Rho GTPases, which alter the endothelial barrier function. An interesting feature in this regulation is that Rho GTPases often have both barrier-protecting and barrier-disturbing functions. While Rac1 enforces the endothelial junctions, it becomes part of a barrier-disturbing mechanism as activator of reactive oxygen species generating NADPH oxidase. Similarly RhoA is protective under basal conditions, but becomes involved in barrier dysfunction after activation of ECs by thrombin. The challenge and promise lies in unfolding this complex regulation, as this will provide leads for new therapeutic opportunities.

\section{Keywords}

Cell-cell interactions, cytoskeleton, endothelial cells, GTPases, protein function / activity

\section{Correspondence to:}

Geerten P. van Nieuw Amerongen

VU University Medical Center

Institute for Cardiovascular Research

Department for Physiology

van der Boechorststraat 7

1081 BT Amsterdam, the Netherlands

Tel.: +3120444 1748, Fax: +31204448255

E-mail: nieuwamerongen@vumc.nl
Financial support:

G.P.v.N.A. is supported by The Netherlands Heart Foundation (The Hague, grant 2003T032). Our laboratory was supported by the EU (EVGN contract LSHM-2003-503254).

Received: June 25, 2009

Accepted after minor revision: August 26, 2009

Prepublished online: September 30, 2009

doi:10.1160/TH09-06-0403

Thromb Haemost 2010; 103: 40-55

\section{Introduction}

During the late 1990s evidence accumulated that the small GTPase RhoA plays an essential role in many vascular pathologies including hypertension, vasospasm and vascular leakage (1-4). Although RhoA was known to regulate many cellular processes including F-actin cytoskeleton and microtubule (MT) dynamics, cell polarity, gene regulation, as well as cell-cycle progression, it remained unclear how alterations in activity of this single molecule could lead to such diverse (biological) activities $(5,6)$. Soon it became clear that a close interplay between RhoA and other members of the Rho GTPase family contribute to the fine-tuning of the biological effects of Rho GTPase activation. Rho GTPases belong to the Ras superfamily of proteins. So far, at least 20 members of the Rho superfamily have been identified $(4,7,8)$. The best-studied members of the Rho GTPase family are RhoA, Racl and Cdc42. They contribute to many aspects of cell behaviour, such as cell migration, cell division and contraction (5). This is reflected in a var- iety of biological processes, such as axon growth, leukocyte infiltration and angiogenesis $(5,6)$. For example, regulation of endothelial barrier integrity, which is disrupted in many vascular pathologies, not only involves RhoA, but also Racl and Cdc42 (9-12). Additionally, it was recognised that the individual Rho GTPases can exert both positive and negative effects on the endothelial barrier integrity, depending on their cellular context and mode of (pathological) activation, adding an additional level of complexity to fine-tuning of the biological response $(13,14)$.

To date, it has been generally accepted that the combined actions of the Rho GTPases require a crucial spatio-temporal regulation of their activities. This regulation, which involves over 150 regulatory proteins, plays a significant role in many vascular pathologies. However, knowledge regarding the contribution of individual regulatory proteins to vascular dysfunction is still fragmental. Yet it is here, where a new area for specific therapeutic targeting opens up. 
Figure 1: Time profiles of RhoA, Rac1 and Cdc42 GTPase activities after stimulation of endothelial cells by thrombin (left) and VEGF (right). Solid lines represent reported Rho GTPase activities, dotted lines represent extrapolated GTPase activities $(17,19-23)$

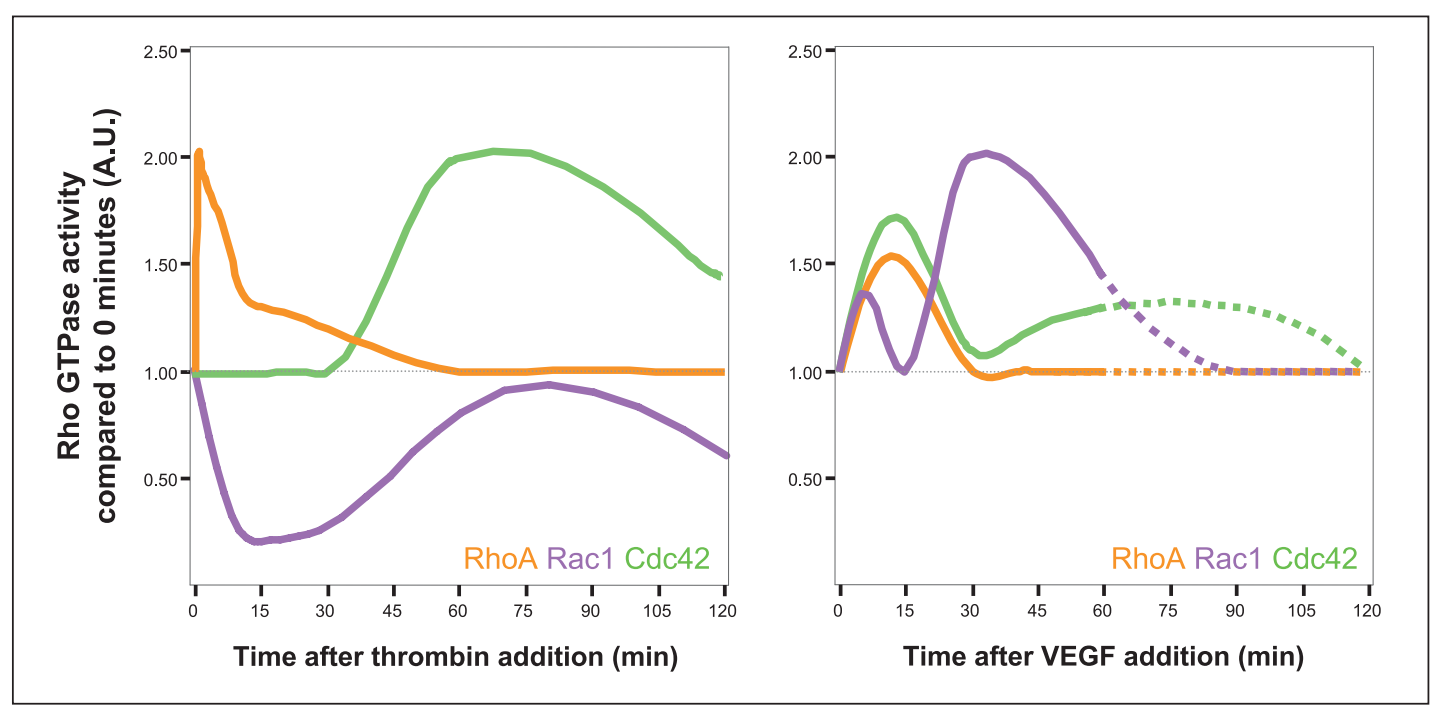

In this review we discuss the principles of regulation of Rho GTPase activity in vascular function. We focus on barrier dysfunction by the hyperpermeability inducers thrombin and vascular endothelial growth factor-A (VEGF) as main representatives of G-protein coupled receptor (GPCR)- and receptor tyrosine kinase (RTK)-mediated Rho GTPase activation, respectively. After a short introduction about the role of Rho GTPases in regulation of the endothelial barrier and the nature of the regulatory proteins involved, we first describe the regulation of Rho GTPases during endothelial barrier maintenance. Next, we describe thrombinmediated Rho GTPase signalling, which results mainly in a contractility and inter-endothelial gap formation. This is followed by a discussion of VEGF-induced Rho GTPase regulation, which disrupts the junctional complexes and causes actin cytoskeleton rearrangement required for endothelial migration. Finally, we briefly discuss Rho GTPase regulation during permeability induced by other agents and indicate some future perspectives.

\section{Ligand-dependent Rho GTPase regulation involved in barrier dysfunction}

The F-actin cytoskeleton plays a major role in the maintenance of the endothelial barrier function, as it determines cell shape, facilitates cell adhesion to the sub-endothelial matrix, and participates in regulation of junctional complexes. The dynamics and structure of the F-actin filaments are mainly regulated by the Rho GTPases RhoA, Cdc42 and $\operatorname{Racl}(7,11)$. In addition, during barrier maintenance the regulation of Racl activity also involves the Rap GTPase Rap1 (15). Several vasoactive agents, like $\beta$-adrenergic agonists, sphingosine-1-phosphate (S1P) $(16,17)$, and adrenomedullin (18), improve the endothelial barrier integrity by enforcing the dense peripheral F-actin band. This process involves changes in the activities of several Rho GTPases. The activities of RhoA, Rac1, Cdc42 and Rap1 are not only involved in regulating barrier main- tenance, but also in active enforcement and disruption of barrier integrity. Other vasoactive agents, such as thrombin and VEGF, can mediate barrier dysfunction via the regulation of often the same Rho GTPases, but activated in another context within the cell.

A reconstruction of the time courses of thrombin- and VEGFmediated alterations in Rho GTPase activities based on several EC studies is shown in Figure 1 (17, 19-23). It is clear that the time course - and for Racl also the direction - of the activity changes are different after activation by these mediators. The profound increase of RhoA induced by thrombin is primarily involved in stress fiber (SF) formation and cell contraction, whereas VEGF-regulated Rho GTPases cause protrusions and cell migration. Simultaneously thrombin also weakens the junctional complexes by indirectly inhibiting Rac1 and relocating Cdc42 (20). In vitro as well as in vivo this results in a hyperpermeable endothelial monolayer (24). In vitro the delayed activation of Racl and Cdc42 restores the endothelial barrier after approximately two hours by initiating cell junction reassembly (20). In contrast, the combined activity changes of Racl, Cdc42 and RhoA induced by VEGF cause cell protrusion and migration (5). Unlike thrombin, VEGF induces rapid activation of Rac1 and the therewith-coupled generation of reactive oxygen species (ROS), leading to endothelial barrier dysfunction (25-27). VEGF-mediated activation of Cdc42 and RhoA is involved in SF formation (28). These data thus show ligand-dependent regulation of the Rho GTPases, and indicate that Rho GTPases under different conditions can have distinct biological effects. This points to contextual activation of downstream targets.

\section{Regulation of Rho GTPase activities}

To appreciate the role of Rho GTPases in health and disease it is necessary to understand how they are regulated. GTPases cycle between an active, GTP-bound state and an inactive, GDP-bound state (Fig. 2). The activation of Rho GTPases requires guanine 


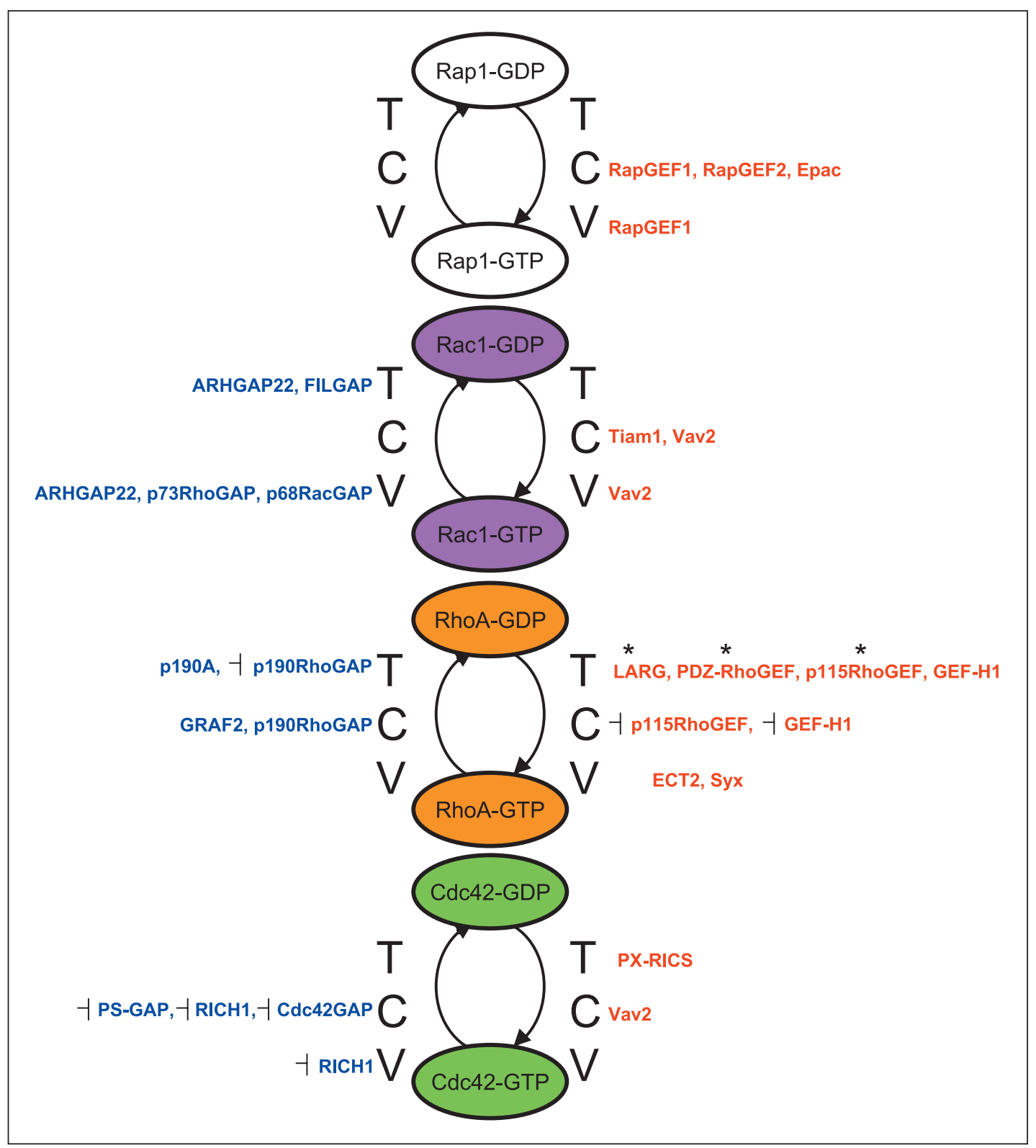

Figure 2: Regulation of GTPases Rap1, Rac1, RhoA and Cdc42 by currently known GAPs (blue) and GEFs (red) during barrier maintenance $(\mathrm{C})$ and thrombin (T)- or VEGF (V)mediated endothelial permeability. * Indicates G-protein binding GEFs, - indicates negative regulation. (For a list of all currently known RhoGEFs and RhoGAPs we refer to supplemental data of Sanz-Moreno et al. (37). Thrombin also activates Ras GTPases, see (38).

nucleotide exchange factors (GEFs) that catalyse the exchange of GDP for GTP in the switch regions of the Rho GTPase. Inactivation of Rho GTPases is mediated by GTPase activating (GAP) factors. The type of nucleotide (GDP or GTP) that is bound at the switch region of the GTPase determines the conformation of the switch region as well as the GTPase activity. Furthermore, guanine dissociation inhibitors (GDIs) subsequently keep Rho GTPases in their inactive state (see below). For more detailed information about small GTPase signalling and their domain functions we refer to Siderovski \& Willard (29) and Rossman et al. (30), respectively. Here, we highlight some characteristics that are important to understand the function of RhoA, Racl and Cdc42 in the regulation of permeability.

\section{Activation of Rho GTPases by guanine nucleotide exchange factors (GEFs)}

The human genome contains at least $83 \mathrm{GEFs}$ that fall into two distinctive families (31). The first is the diffuse B-cell lymphoma (Dbl)-family. Members of this family contain a Dbl homology (DH) domain followed by a pleckstrin homology $(\mathrm{PH})$ domain. The DH domain mediates RhoGEF activity, while the PH domain has an auto-inhibitory role (30-33). The $\mathrm{PH}$ domains of these typical RhoGEFs interact with phospholipids and may therefore localise the GEF and subsequent GTPase activation to the membrane. A specific subgroup of these RhoGEFs provides a structural motif known as regulator of G-protein signalling (RGS) domain, by which they can form molecular complexes with $G \alpha_{12 / 13}$ units of GPCRs. This interaction accelerates the GTPase activity of $\mathrm{G \alpha}_{12 / 13}$ 
5- to 10-fold, thus enhancing the signal-to-noise ratio of RhoA activation by $\mathrm{G} \alpha$ proteins. Known members of this sub-group ( ${ }^{*}$ in Fig. 2) are leukemia-associated RhoGEF (LARG, also known as ARHGEF12), p115RhoGEF (also known as GEF1 and ARHGEF1) and PDZ-RhoGEF (also known as GTRAP48 and ARHGEF11) (29, 34-36).

The second typical RhoGEF family is the DOCK-family. This family lacks DH and PH domains. RhoGEF activity is instead mediated by DOCK homology region (DHR) 1 and 2 domains (39). DOCK-family members activate either Rac1 or Cdc42 (39-41). To date no member of this family has been associated with RhoA activation.

There are several mechanisms for GEF activation. The first is by phosphorylation. It has e.g. been shown that protein kinase $\mathrm{C} \alpha$ (PKC $\alpha$ ) activates $\mathrm{p} 115 \mathrm{RhoGEF}$ via a serine phosphorylation (25, 42 ), while tyrosine phosphorylation of PDZ-RhoGEF and LARG was shown by focal adhesion kinase (FAK) $(42,43)$. More importantly, GEFs can be activated by RTKs via Src and adaptor proteins. Of the 58 human RTKs, more than half are known to activate at least one of 16 RhoGEFs in addition to activating their common signal transduction pathways via phosphatidylinositol 3-kinase (PI3K), phospholipase $\mathrm{C}$ (PLC) and the mitogen-activated protein kinase (MAPK) pathways (44).

Finally, activation of GEFs may be dependent on their subcellular localisation. This mechanism is known to regulate the spatiotemporal activation of RhoA and Rac1. Examples are ECT2 and Net1 (also known as ARHGEF8 and NET1A). These are nuclear RhoGEFs, but their translocation to the cleavage furrow and the plasma membrane, respectively, has been associated with RhoA activation $(45,46)$. In addition, the activation of Rac1 was associated with plasma membrane localization of the cytosolic RhoGEF Tiam1 $(30,47)$. The RhoGEF GEF-H1 (also known as ARHGEF2, GEF, p40, LFP40) interacts with MTs and is involved in the crosstalk between the MT- and actin-network $(48,49)$. MT interaction inhibits its GEF activity for RhoA at the plasma membrane, but alternatively may switch its GEF specificity towards $\operatorname{Rac} 1(30,50)$.

\section{Inactivation of Rho GTPases by GTPase activating proteins (GAPs)}

The second group of molecules to regulate Rho GTPase activity are the GAPs. In contrast to what their name suggests, Rho GTPases have a relatively slow intrinsic GTPase activity. GAPs enhance the GTP hydrolysis of the active GTPases thereby causing inactivation of these proteins (Fig. 2). To date, more than 70 RhoGAPs have been identified. Interestingly, at least one of them, p73RhoGAP, has a preferential endothelial expression $(51,52)$, but expression of most GAPs is ubiquitous. Similar to RhoGEFs, RhoGAPs are also regulated by extracellular cues, such as growth factors.

Several mammalian RhoGAPs have been implicated in specific Rho GTPase-mediated biological functions such as angiogenesis, migration, cell blebbing and endocytosis. As nicely surveyed by Tcherkezian and Lamarche-Vane (53), studies of the past decade have shed some light on the question why the RhoGAPs outnumber Rho GTPases by 2 - to 3 -fold $(53,54)$. Probably more important than tissue-specificity, is that GAPs, but also GEFs, may selectively regulate a specific downstream signalling pathway by activating Rho GTPase activities when they are in complex with other proteins, thereby leaving other GTPases and their pathways unaltered. Some RhoGAPs seem to specifically act on a single Rho GTPase, whereas others display activity towards multiple Rho proteins, explaining why Rho activities simultaneously rise or fall. Furthermore, RhoGAPs may act as scaffold molecules independent of their GAP activity, mediating cross-talk between Rho GTPases and other signalling pathways. In addition to GAP and GEF domains, these Rho GTPase regulatory proteins often also have docking sites for other proteins so that they integrate signals from different pathways thereby directing specific downstream signalling.

\section{Rho GTPase regulations by guanine nucleotide dissociation inhibitors (GDIs)}

During nucleotide exchange GTP is preferentially bound to Rho GTPases because intracellular levels of GTP are substantially higher than those of GDP. GDIs interact with the switch domains of Rho GTPases to prevent activation by GTP and lock Rho GTPases in their inactive state. Furthermore, GDIs retain inactive GTPases in the cytosol by shielding off their membrane tag (30). There are currently three RhoGDIs known. RhoGDI $\alpha$ (also known as RhoGDI-1, Rho-GDI $\alpha$, RABGD1A and RABGDIA) is ubiquitiously expressed, whereas RhoGDI $\beta$ (also known as D4, Gdid4, Ly-GDI, ArhGDI $\beta$ ) is expressed in haematopoetic cells and RhoGDI $\gamma$ (also known as Rho-GDI3 and ARHDIAG) is preferentially expressed in organs and the brain, where it is anchored to the membrane $(55,56)$. Although the mechanisms are still largely unknown, RhoGDIs seem to regulate the fine-tuning of Rho GTPase activities via their association with multiprotein complexes in particular membrane microenvironments $(55,56)$.

\section{Basal maintenance of the endothelial barrier}

Initial attention for Rho GTPases was drawn to RhoA activation and its role in cell contraction and endothelial hyperpermeability (see section 3). During the last years the focus has moved to the role of Rac and Cdc42 in the assembly and stability of inter-endothelial junctions $(20,57)$. Furthermore, an additional role for RhoA and its effector Rho kinase in barrier maintenance was recognised (20).

Both tight junctions (TJs) and adherens junction (AJs) contribute to the intercellular sealing of the endothelial monolayer and the resulting endothelial barrier function. In contrast to blood-brain ECs, in which the TJs form a sealing belt, TJs in most other ECs have a mosaic structure leaving space for the passage of macromolecules. The AJs contribute largely to the barrier proper- 


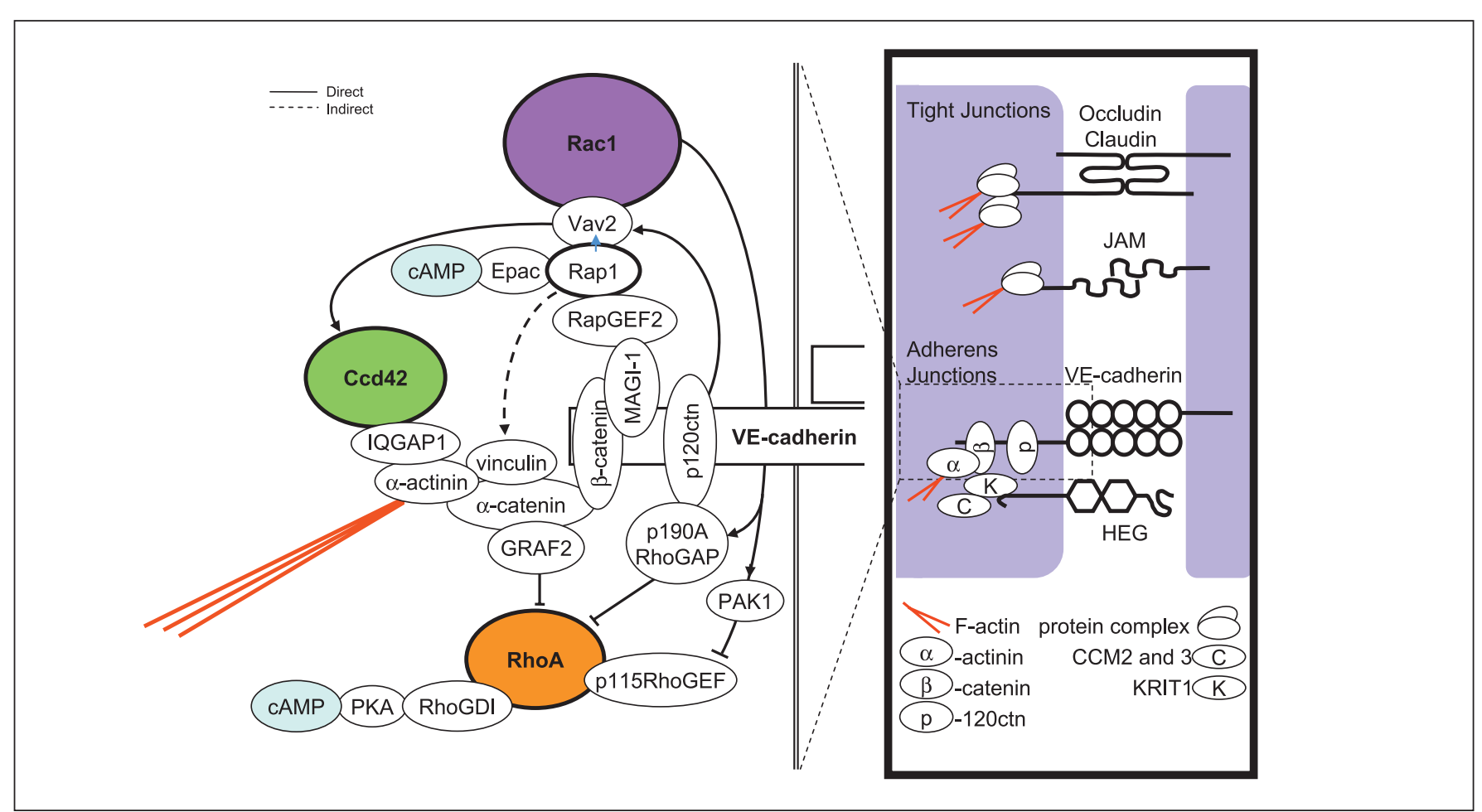

Figure 3: Rho GTPase activity regulates barrier integrity. The major TJ and $A J$ protein interactions are indicated in the right box. On the left side part of an $\mathrm{AJ}$ is enlarged. Here, cadherin engagement with the catenins via Vav2 activates Cdc42 and Rac1. VE-cadherin bound p120ctn and Rac1 inhibit RhoA by activation of p190RhoGAP. Via PAK1-mediated inhibition of

ties of these latter endothelia in interaction with the actin cytoskeleton ( Fig. 3)

\section{Proteins and assembly of AJs}

Much attention has been given to the regulation of AJs and their pivotal protein VE-cadherin, a membrane spanning protein that forms homotypic interactions between adjacent ECs (25) (Fig. 3, right panel). The intracellular part of VE-cadherin is connected to the F-actin cytoskeleton via both $\alpha$-and $\beta$-catenin (25). In addition, a third catenin, p120catenin (p120ctn), binds to VE-cadherin (58). P120ctn binding to VE-cadherin is an important step in the regulation of AJ stabilisation as well as in Rho GTPase regulation.

Racl and Cdc42 are activated at sites where junctional complexes are formed $(59,60)$, while RhoA activity is downregulated when the monolayer reaches confluence (61). Indeed, Rac1-deficient ECs are unable to form lamellipodial structures, focal adhesions and cell-cell contacts (62). Furthermore, many GTPase regulator activities are directly initiated by cadherin engagement (see below). Thus, a precise spatial and temporal fine-tuning of the activity of Rho family GTPases is critically important in the establishment and maintenance of junctions.
p115RhoGEF Rac1 activity further suppresses RhoA. Linking of the junctional complex to the actin cytoskeleton stabilizes the AJs and further inhibits RhoA by activation of GRAF2. Enhanced levels of cAMP induce Rap1-mediated Rac1 activation as well as RhoGDI-mediated inhibition of RhoA. For further details see text.

Another AJ protein is the endothelial-specific HEG (heart of glass) transmembrane receptor (Fig. 3, right panel), which interacts with an as yet unknown extracellular ligand to activate a coordinated signalling response through inhibiting the activity of RhoA. This response requires the cerebral cavernous malformations proteins CCM1 (or KRIT1), CCM2 and CCM3 (63) and leads to a cellular response that may include induction of endothelial vacuolisation and homotypic endothelial cell-to-cell junction formation. These cellular events are required for normal vascular development and maintenance of vascular integrity. Defects in these steps result in enhanced activation of RhoA and may lead to the pathological changes associated with hereditary cerebral cavernous malformations in humans $(64,65)$. Loss of RhoA degradation, which is achieved by the ubiquitin ligase (E3) Smurf-1, after interaction with CCM2 is accompanied by increased endothelial permeability $(66,67)$. Deletion of CCM2 did not affect the Racl concentration in ECs (66).

\section{Interaction of junctional proteins with Rho GTPases}

Concerning basal endothelial barrier regulation, much attention has been given to the relationship between VE-cadherin, the catenins and Rho GTPases. After cadherin engagement p120ctn bind- 
ing activates Vav2, a GEF for Cdc42 and $\operatorname{Rac1}(68,69)$ (Fig. 3, left panel). This GEF positively regulates $\mathrm{Cdc} 42$ and Racl activity. Both GTPases bind IQGAP (IQ motif containing GTPase-activating protein 1). Unlike the name suggests IQGAP1 does not have GAP activity, meaning that it does not enhance the GTP hydrolytic activity of the GTPases. Instead its binding to Cdc42 and Racl inhibits their intrinsic GTPase activity, thus keeping them in their active, GTP-bound state (70-72). By recruiting IQGAP1, Rac1 and Cdc42 inhibit the interaction of IQGAP1 with $\beta$-catenin thereby initiating the binding of $\alpha$ - and $\beta$-catenin $(20,73)$. This cross-links the complex with the actin cytoskeleton and stabilises the AJ (74). In contrast, inactivation of Rac1 and Cdc42 causes dissociation from IQGAP1 that then binds to $\beta$-catenin at the AJ complex. As a result $\alpha$ - and $\beta$-catenin dissociate, leading to weaker adhesion and inter-endothelial gap formation (75).

It is likely that similar mechanisms also occur at the TJ level. Recently, data revealed that in epithelial cells RICH1 (also known as ARHGAP17), which has GAP activity for Cdc42 is required for the stability of epithelial TJs. In vivo the TJ component and scaffolding protein angiomotin binds RICH1. This suppresses its GTPase activity, thus allowing Cdc42-mediated barrier protection (76).

\section{RhoA activity and gap closure}

Consistent with previous data in other cells (77), we recently found that in non-stimulated EC monolayers RhoA activity, as measured by Raichu-FRET, occurs prior to gap closure (unpublished data). The inactivation of RhoA upon gap closure was explained by binding of p120ctn to the newly formed AJ complex, which causes p120ctn-mediated activation of p190RhoGAP and thus inactivation of $\operatorname{RhoA}(58,78)$ (Fig. 3, left panel).

Another molecular mechanism has been described that reduces RhoA activity upon establishment of AJs. ARHGAP10 (also known as GRAF2) in vitro inactivates RhoA and Cdc42 (53). In vivo human GRAF2 inhibits RhoA and recruits $\alpha$-catenin to the newly formed cell-cell contacts (79) (Fig. 3), thereby stabilising the AJs.

\section{Inactivation of RhoA by Rac1}

Under confluent conditions, cAMP stimulates Epac which has GEF activity for Rap1 (15). Rap1 initiates the activation of Vav2 resulting in active Rac1 (80). Activated Rac1 further inactivates RhoA by several downstream targets (Fig. 3). Rac1 subsequently activates p190RhoGAP thereby inhibiting RhoA (58). Furthermore, Rac1 activates its downstream target PAK1, which inhibits p115RhoGEF, thus further repressing the activity of $\operatorname{RhoA}(24,81)$. In addition, enhanced cAMP levels activate protein kinase A (PKA), which stimulate RhoGDI binding - and thus further inactivation - of RhoA (82).
An additional pathway for Rapl activation was shown upon VE-cadherin engagement with $\beta$-catenin (Fig. 3 ). The membraneassociated guanylate kinase with inverted domain structure-1 (MAGI-1), which links via $\beta$-catenin to VE-cadherin $(83,84)$, binds RapGEF2 (also known as PDZ-GEF1, RA-GEF, NRAPGEP, Rap-GEP and CNrasGEF) (84). The subsequently activated Rap1 recruits vinculin from cell-matrix to cell-cell contacts (83). Vinculin supports the linking of $\alpha$-catenin to $\alpha$-actinin, thereby further stabilising VE-cadherin-based cell adhesion (85). Rap1 thus not only mediates the activation of Racl but also promotes the stabilisation of VE-cadherin based junctions.

Finally, it has been suggested that activation of Rac1, which is accompanied by the release of RhoGDI $\alpha$, induces the inhibition of RhoA activity by increasing the interaction of RhoA with the newly released RhoGDI $\alpha$ (86).

\section{Summing up}

In conclusion, the maintenance of the endothelial barrier depends on RhoA, Cdc42 and Racl activation. RhoA activity decreases upon gap closure due to VE-cadherin and p120ctn engagement. The binding of the AJs to the actin cytoskeleton involves Cdc42, which is kept in its active state by binding to IQGAP1. The linking of the AJs to the actin cytoskeleton also activates GRAF2, which inhibits RhoA. The rise in cAMP levels, via PKA, activates RhoGDI $\alpha$ thereby inhibiting RhoA. In addition, rising cAMP levels and cadherin engagement induce the activation of Epac and RapGEF2, respectively. These GEFs stimulate Rap1, which via Vav2 activates Rac1. Racl activation results in the inhibition of p115RhoGEF and thus a suppression of RhoA. Vav2 activity is further enhanced by p120ctn binding to the junctional complexes. The latter also directly activates p190RhoGAP thereby further inhibiting RhoA activity.

\section{Receptor-mediated cell activation by thrombin}

One of the best-studied activators of RhoA in the context of endothelial permeability regulation is thrombin. Thrombin mediates its effect on RhoA directly via its receptor PAR-1 $(87,88)$. Thrombin-mediated cleavage of PAR-1 activates several heterotrimeric G-proteins and their subsequent signalling pathways. The release of the $\alpha$-subunits of these G-proteins $\left(\mathrm{G \alpha}_{11 / \mathrm{Q}}, \mathrm{G} \alpha_{12 / 13}\right.$ and $\left.\mathrm{G \alpha}_{\mathrm{i}}\right)$ regulates the activity of $\operatorname{RhoA}(35,89-91)$ and $\operatorname{Racl}(15,80)$, either directly $(35,91)$, or via intracellular $\mathrm{Ca}^{2+}(35,89,91)$ or cyclic adenosine monophosphate (cAMP) elevation $(15,80,82,90)$. As recently shown, knockdown of $\mathrm{G} \alpha_{11 / \mathrm{Q}}$ or $\mathrm{G} \alpha_{12 / 13}$ in human ECs prevents RhoA activation by thrombin $(92,93)$, indicating that both pathways are highly interrelated at the level of RhoA activation and together control downstream signalling leading to EC contraction and permeability. 


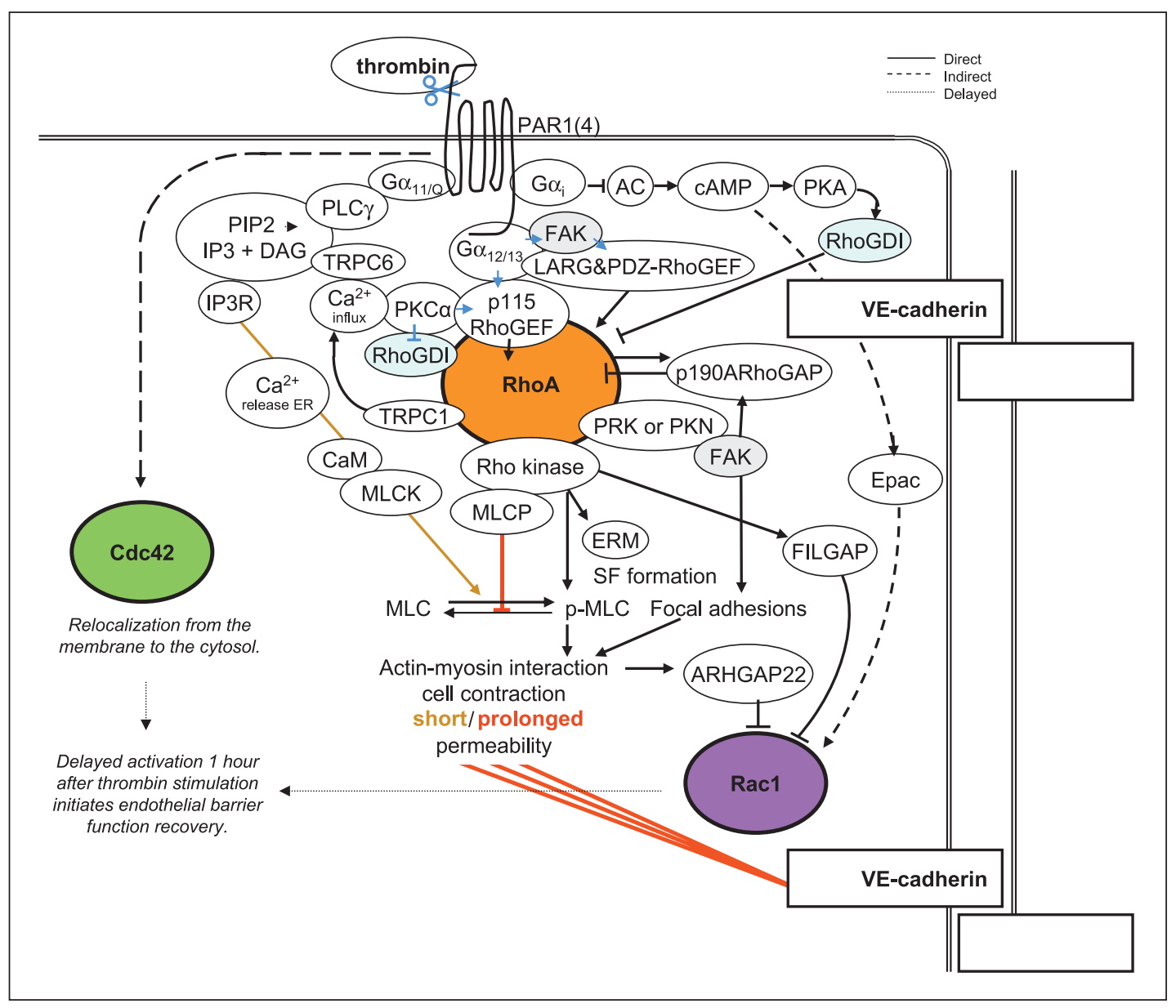

Figure 4: Thrombinmediated regulation of Rho GTPases involves several G-proteinactivated pathways. For details see text. The inhibitory effects of Rac1 on RhoA activity are discussed in section 3 "Modulation of Rac1 activity by thrombin" and depicted in Figure 3.

\section{Direct and indirect (in) activation of Rho GTPases by thrombin}

In the following chapter we survey the thrombin-mediated activation of RhoA, which involves several pathways and is associated with SF formation and cell contraction. Next, we discuss how thrombin may prolong RhoA activity. Furthermore, it is explained how thrombin initially causes an inhibition of Rac1, which is implicated in weakening of the junctional complexes, while later, a delayed recovery of both Racl and Cdc42 activities results in endothelial barrier recovery.

\section{G-protein-mediated activation of RhoA by thrombin}

Thrombin-mediated cell contraction requires two pathways that are interrelated at the level of RhoA activation ( Fig. 4). The first pathway involves thrombin-mediated activation of $\mathrm{G} \alpha_{11 / \mathrm{Q}}$, which causes coupling to phospholipase $\mathrm{C} \gamma(\mathrm{PLC} \gamma)$ that in turn generates inositol trisphosphate (IP3) and diacylglycerol (DAG). IP3 via its receptor on the endoplasmic reticulum (ER) liberates $\mathrm{Ca}^{2+}$ from the ER. This $\mathrm{Ca}^{2+}$ release activates calmodulin $(\mathrm{CaM})$, which phosphorylates myosin light chain kinase (MLCK) resulting in enhanced myosin light chain (MLC) phosphorylation (Thr18 and Ser19) (94-96). Increased MLC phosphorylation enhances acto- myosin interactions (97), cell contraction and permeability (98). Meanwhile DAG activates the transient receptor potential channel (TRPC)-6 at the plasma membrane, which allows for $\mathrm{Ca}^{2+}$ influx from the extra-cellular matrix and activation of PKC $\gamma$ (99). PKC $\gamma$ has a dual role. It directly mediates the inhibitory phosphorylation (Ser96) of RhoGDI $\alpha(100,101)$ and the stimulatory phosphorylation of p115RhoGEF $(91,101)$. This causes RhoA liberation and activation, respectively.

The second pathway involves the thrombin-mediated activation of $\mathrm{G \alpha}_{12 / 13}$, which binds and activates $\mathrm{p} 115 \mathrm{RhoGEF}$, thus resulting in activated RhoA $(34,102)$. Via its downstream target Rho kinase, RhoA directly enhances SF formation and cell contraction. Rho kinase induces the phosphorylation of the regulatory subunit MYPT1 (Thr691 and Thr855) of the myosin light chain phosphatase (MLCP) (103). This decreases the phosphatase activity of MLCP, thereby reducing the de-phosphorylation of $\operatorname{MLC}(13,104)$. Rho kinase by itself also can phosphorylate MLC (Ser19) (105). Thereby further enhancing MLC phosphorylation, which leads to acto-myosin interactions as described above.

\section{Feedback mechanisms prolonging RhoA activation}

Several positive feedback mechanisms can prolong the activation of RhoA and the subsequent cell contraction (Fig. 4).Via the mem- 
brane localised TRPC-1, RhoA further increases the $\mathrm{Ca}^{2+}$ influx (25) that prolongs the $\mathrm{G} \alpha_{11 / \mathrm{Q}}$-mediated activation of MLCK, PKC $\alpha$ and RhoA. Three additional feedback pathways involve Rho kinase signalling. First, Rho kinase phosphorylates ezrin (Thr567), radixin (Thr564) and moesin (Thr558), which are collectively known as the ERM-proteins (106). ERM phosphorylation enhances SF formation thereby stimulating cell contraction and endothelial permeability. Second, it was shown for endothelin1-stimulated vascular smooth muscle cells that Rho kinase causes an inhibitory phosphorylation (Ser1150) of p190RhoGAP (also known as ARHGAP5, GRLF1, GRF-1) (107) of which two isoforms, p190A and p190B, are known. The first is associated with prolonging RhoA activation (107). As prolonged RhoA activation is also seen during thrombin stimulation, it is likely that Rho kinase-mediated p190A inhibition also occurs during thrombin stimulation of ECs. Third, thrombin/p115RhoGEF-mediated activation of Rho kinase induces MT disassembly. This activates GEF-H1, a RhoA specific GEF that is known to localise at the MTs $(48,49)$. In its MT-bound state, the guanosine-exchange activity of GEF-H1 is suppressed, whereas GEF-H1 released from MTs stimulates GEF activity and may thus induce further activation of RhoA (48).

In addition to Rho kinase, RhoA also activates protein kinase C-related kinase (PRK or PKN), which can regulate FAK-mediated focal adhesions. Rho kinase and PRK-mediated activities are both required for full thrombin-induced EC hyperpermeability (92). Accumulating evidence suggests a dual, time-dependent role for FAK in the regulation of thrombin-mediated RhoA activity: Thrombin-mediated FAK phosphorylation occurs after 5 minutes (min) and peaks after 10 min (43). Approximately 3-5 min after thrombin stimulation $\mathrm{G \alpha}_{12 / 13}$-mediated FAK activation results in an activating phosphorylation of PDZ-RhoGEF and LARG, which causes prolonged RhoA activity (43). Subsequently, 10 min after thrombin stimulation FAK activates p190RhoGAP (108), which in vivo preferentially inhibits RhoA activity (108-110). This time course of p190RhoGAP phosphorylation coincides with the decrease in RhoA activity both in human pulmonary artery and umbilical vein ECs $(21,108)$. Thus, thrombin-mediated FAK activation in ECs first extends and then down-regulates the activation of RhoA (see to Suzuki et al. (111) for more detail about interactions between $\mathrm{G} \alpha_{12 / 13}$ proteins and their RhoGEFs).

Finally, thrombin stimulates RhoA activity by activating $G \alpha_{i}$. The $\mathrm{G} \alpha_{\mathrm{i}}$ inhibits adenyl cyclase (AC), which reduces cAMP levels in vascular smooth muscle cells (112) and in ECs $(90,113)$. Via attenuation of PKA this causes a reduced RhoGDI $\alpha$ activity thereby allowing further activation of RhoA (82).

\section{Modulation of Rac1 activity by thrombin}

As indicated before, thrombin-mediated endothelial permeability in vitro also involves a one hour-lasting inactivation of Racl (93). This is thought to weaken the junctional complexes, which in combination with cell contraction, results in enhanced endothelial permeability. Several thrombin-mediated pathways may be involved in the inhibition of Racl (Fig. 4). First, it is known that the PAR-
$1 / G \alpha_{i}$-mediated inhibition of AC, which decreases cAMP levels $(90,113)$ causes the inhibition of Racl (93), a process that involves the inhibition of the RapGEF Epac and Rap1 (80) (see section 2 "Inactivation of RhoA by Rac1"). Second, in tumor cells the RhoA/ Rho kinase-mediated acto-myosin interaction inhibits Racl via the activation of the usually vascular specific ARHGAP22 (also known as RhoGAP2) (37). Third, Rho kinase activates the vascular specific FILGAP, a longer isoform of p73RhoGAP (also known as ARHGAP24 and RC-GAP72), which also antagonises Rac1 (51). Although no data is available on thrombin-mediated ARHGAP22 or FILGAP regulation in ECs, it has been shown that this Rho-mediated inhibition of Racl regulates amoeboid movement, which depends on Rho/Rho kinase signalling $(37,51)$. It seems likely that thrombin signalling also causes ARHGAP22 and FILGAP activation. It is of interest to note that suppression of Rac1, indirectly enhances p115RhoGEF activity thus further stimulating RhoA activation (24).

Thrombin not only inhibits Rac1, but occasionally may also cause a delayed Racl activation by indirect mechanisms. In mouse pulmonary ECs thrombin-mediated activation of PKC $\alpha$ leads to the activation of sphingosine kinase that catalyses the formation of S1P from sphingosine, thereby causing paracrine activation of the S1P receptor (17). Subsequently, G $\alpha_{\mathrm{i}}$-activated PKCE induces phospholipase D2 activation and enhanced Racl activity in vascular smooth muscle cells (114). Delayed thrombin-mediated activation of Racl stabilises newly formed junctional complexes and enhances the endothelial barrier integrity $(20,73,93)$. Rac1 activity increases during junction formation $(10,60)$. In contrast to other authors, Diebold et al. (115) reported a rapid increase in active Rac1 15 min after thrombin stimulation. This increase, which was accompanied by activation of NADPH oxidase, occurred indirectly via phosphodiesterase 2 .

For more details about the role of Racl in maintaining the endothelial barrier integrity see section 2 "Interactions of junctional proteins with Rho GTPase”.

\section{Cdc42 modulation by thrombin}

Besides Rac1 Cdc42 participates in recovery of the endothelial barrier integrity. Thrombin stimulation of ECs quickly causes the relocalisation, but not inactivation (116), of Cdc42 from the plasma membrane to the cytosol (93). This basal Cdc42 activity seems to contribute to the correct thrombin-induced acto-myosin contraction and SF formation (117). Delayed Cdc42 activation coincides in time with the activation of Racl (1 hour after thrombin stimulation) (93). Cdc42 additionally, is involved in Golgi-derived membrane trafficking $(118,119)$. The cycling of Cdc42 between the active and inactive form seems essential for its function in protein transport and polarity. Cdc42 at the Golgi occurs via PXRICS, a splicing variant of RICS (also known as p250RhoGAP, p200RhoGAP and Grit), which is expressed in a wide variety of cells and has GAP activity for Cdc42. In HeLa cells PX-RICS interacts with $\beta$-catenin and regulates the ER-to-Golgi transport of the $\mathrm{N}$-cadherin/ $\beta$-catenin complex (120). In epithelial cells the E-cadherin recycling to the membrane is regulated by both Racl and 
Cdc42 and takes place prior to p120catenin binding to the junctional complexes (see section 4) (121).

\section{Summing up}

By activation of its receptor PAR-1 thrombin mediates activation of RhoA via $\mathrm{G} \alpha_{11 / \mathrm{Q}^{-}}, \mathrm{G} \alpha_{12 / 13^{-}}$and $\mathrm{G} \alpha_{\mathrm{i}^{-}}$-mediated pathways. The availability of RhoA for activation is enhanced by $\mathrm{G \alpha}_{11 / \mathrm{Q}} / \mathrm{Ca}^{2+} /$ PKC $\alpha /$ - and $G \alpha_{i} / c A M P / P K A-m e d i a t e d ~ R h o G D I \alpha$ inhibition. The activation of RhoA is induced by $\mathrm{G}_{11 / \mathrm{Q}} / \mathrm{Ca}^{2+} / \mathrm{PKC} \alpha$ and G $\alpha_{12 / 13}(\mathrm{FAK})$-activated RhoGEFs (p115RhoGEF, LARG and PDZ-RhoGEF). Subsequent inactivation of RhoA is mediated by FAK-activated p190(A)RhoGAP. Thrombin stimulation further inhibits Rac1, partially by decreased cAMP levels and partially due to Rho kinase-mediated activation of FILGAP and acto-myosin interaction-induced activation of ARHGAP22. Finally, thrombin causes a delayed activation of both Rac1 and Cdc42. Cdc42, which at the Golgi is activated by PX-RICS, stimulates the transport of junctional proteins towards the plasma membrane, thereby causing the repair of the junctional complexes.

\section{Consequences for the regulation of permeability}

Infusion of thrombin receptor activating peptide, which mimics the action of thrombin, was shown to induce pulmonary microvascular permeability in situ (122). Its infusion into the hindleg of a heparinised rat causes a rapid swelling due to increased endothelial permeability (unpublished data). Endothelial permeability induction by thrombin in endothelial cell monolayers in vitro has extensively been used to study mechanisms underlying permeability regulation (123). This enhancement occurs in a biphasic way. Initially a rapid drop in transendothelial electrical resistance occurs accompanied by a moderate increase in permeability for macromolecules like albumin and peroxidase. This is accompanied by Racl inhibition and Cdc42 translocation, which facilitate loosening of the AJs. Together with the thrombin-induced activation of tyrosine kinases like $\operatorname{Src}(21,25)$, which by phosphorylational changes reduces the homotypic interaction between junctional proteins $(98,124)$, this causes the disruption of the AJs $(125)$. At the same time RhoA/Rho kinase activity together with $\mathrm{Ca}^{2+} / \mathrm{MLCK}$ activity enhance actin-myosin interaction, induce SFs and cause a general cell contraction. The latter mechanism causes a second phase of a further increased hyperpermeability, which starts 10-15 min after thrombin exposure and ceases after $60 \mathrm{~min}$, when Racl and Cdc42 activities also normalise again. Overall this second phase leads to inter-endothelial gap formation and cell rounding. While cell contraction was already implied in early studies on endothelial permeability in vivo (126), the extent of contraction and leakage in vitro seems disproportional as compared to the situation in vivo. In situ thrombin stimulation of arteriolar and venular ECs indeed leads to cell contraction and cell rounding, but the inter-endothelial gaps are clearly smaller (127). In ECs in vivo RhoA/Rho kinase activation only leads to a contraction at the cell margins, which is nonetheless enough to cause vascular leakage (128). An increasing amount of evidence suggests that this apparent difference in degree of cell contraction is at least partially due to the flexibility of the underlying tissue.

Interestingly RhoA/Rho kinase activity, which is present at the cell margins, disappears after thrombin stimulation, while then, Rho kinase activity at the SFs becomes visible (127). This local decrease at the cell margins, together with altered local activities of Rac1 and Cdc42 mentioned above, point to potential roles of Rho GTPases in the formation and maintenance of junctional complexes and endothelial barrier function.

Finally, when primed e.g. during the late phase of sepsis or upon activation with activated protein C, PAR1 switches from being a vascular-disruptive receptor to a vascular-protective receptor, a phenomenon that might involve transactivation of PAR2 and S1P receptor-1 (129-131). This 'role reversal' of PAR-1 is accompanied by a dampening of RhoA activation and recovery of Rac-GTP levels.

\section{VEGF receptor-mediated activation involved in permeability}

VEGF-mediated endothelial permeability is complex and involves the formation of transendothelial pores through the fusion of endothelial vesicles and disruption of endothelial junctions. Though the precise mechanisms are still unclear, Rho GTPases are also involved in this latter aspect of VEGF-mediated permeability (11). Compared to thrombin, VEGF-mediated Rho GTPase activation appears more restricted to a direct effect on the disruption of the AJs.

ECs express two VEGF receptors that recognise VEGF-A, known as VEGFR1 (also known as Flt-1) and VEGFR2 (known as Flk1 and KDR). They belong to the family of receptor tyrosine kinases (RTKs). Though during embryogenesis both receptors are involved, in adult ECs VEGFR2 is in particular involved in activating ECs during angiogenic processes. Upon binding to VEGF, VEGFR2 undergoes dimerisation and oligomerisation, which activates its intrinsic tyrosine kinase activity resulting in auto- and trans-phosphorylation on specific tyrosine residues in the cytoplasmic domain (132). These tyrosine residues, when phosphorylated, are docking sites for Src homology 2 (SH2) binding domain containing downstream effectors. For more detailed information on VEGFR2 phosphorylation see Olsson et al. (133).

Under confluent conditions in vitro and in vivo, VE-cadherin interacts with VEGFR2 $(10,134)$ in an IQGAP1-mediated manner (135). While the number of these complexes increase upon VEGF stimulation (135), VEGF, in a Racl-dependent manner, leads to vascular permeability $(26,136)$. VEGF-mediated RhoA and Cdc42 activation are involved in cytoskeleton rearrangements needed for cell migration (22), a process that requires binding of activated VEGFR2 to the $\alpha v \beta 3$ integrin (137). 


\section{VEGF/RTK-mediated Rac1 activation and ROS generation}

During activation by VEGF, the VEGFR2 is phosphorylated on multiple tyrosine residues, including Tyr951, Tyr1175, Tyr1212 and Tyr1214. While Tyr1175 is essential for PLC $\gamma$-mediated ERK1/2 activation and DNA synthesis (138), and Shb-mediated focal adhesion formation (133), Tyr1212, Tyr1214, and probably Tyr951, are involved in activation of Rho GTPases.

As a result of VEGF-induced phosphorylation of VEGFR2 (Tyr1212) Src is activated (139). Within 3 min this stimulates Vav2, Rac1 and its downstream target PAK1 $(23,140)$. PAK1 phosphorylates VE-cadherin (Ser665) inducing the recruitment of $\beta$-arrestin2 and the internalisation of VE-cadherin into clathrin-coated vesicles (141) (Fig. 5). Deletion of Rac1 in vitro in primary ECs indeed decreased VEGF-mediated endothelial permeability (62). This observation contrasts to other observations that Rac is required for the generation of the endothelial barrier under basal conditions $(59,60,62)$. This suggests different modes of action of $\mathrm{Racl}$ in regulating endothelial permeability.

VEGF-activated Rac1 can also activate NADPH oxidase at the EC membrane. Indeed, in human pulmonary microvascular ECs it was demonstrated that VEGF-mediated activation of Racl caused ROS generation that was inhibited by a NADPH oxidase inhibitor (26). This activation was accompanied by an increase in endothelial permeability, which was based on the phosphorylation of Tyr658 and Tyr731 of VE-cadherin, which cause detachment of p120ctn and $\beta$-catenin, respectively, and phosphorylation of Tyr654 on $\beta$-catenin. Inhibition of either Racl or NADPH oxidase activity prevented the increase in endothelial permeability and the accompanying phosphorylations of VE-cadherin. Whether ROS act directly on the kinase activity, or, more likely, inhibit VE-cadherin associated tyrosine phosphatases, has still to be clarified. Furthermore, the phosphorylations of VE-cadherin may occur contextually. It is unknown whether phosphorylation of VE-cadherin at Tyr658 and Tyr731 will occur simultaneously with that of Ser665 or that they exclude each other mutually. Similarly, the phosphorylation of Tyr685 of VE-cadherin may affect the other phosphorylations (142).

ROS production is apparently an essential component in signalling cascades that mediate Rac1/p190RhoGAP-induced downregulation of RhoA (27). ROS production causes p190RhoGAP translocation to the AJs, where it binds p120ctn, and subsequently inhibits local RhoA activity (58). It thus plays a role in the stabilization of cell-cell contacts (Fig. 5). A regulatory role in this process was shown for IQGAP1, which upon VEGF stimulation is rapidly $(<5 \mathrm{~min})$ recruited to VEGFR2. This is associated with tyrosine phosphorylation of IQGAP1 and subsequent binding and activation of Rac1. In addition, it causes further VEGFR2 association with the VE-cadherin/ $\beta$-catenin complex (135). Deletion of IQGAP1 indeed decreases Rac1-mediated ROS production and prevents cell migration and proliferation $(135,143)$.

\section{Additional effects of VEGF/RTK on RhoGTPases}

In addition to the Tyr1212 phosphorylation, phosphorylation of the adjacent Tyr1214 on VEGFR2 causes binding and activation of heat-shock protein 90 (HSP90). HSP90 indirectly mediates the activation of RhoA and subsequently Rho kinase, which, as described above, leads to acto-myosin interaction and cell contraction (144). Rho kinase also phosphorylates FAK (Ser732). This specific phosphorylation is a crucial step for subsequent additional phosphorylations of FAK by Src (144) and Shb $(144,145)$, which are crucial for paxillin and vinculin recruitment to the focal adhesion sites (144).

The transient Tyr1214 phosphorylation of VEGFR2 furthermore recruits and activates the adapter protein Nck, which triggers the Src family kinase Fyn, indirectly (maybe via PAK2) resulting in Cdc42 activation (144). Cdc42 targets PAK1 which turns on SAPK2 (stress-activated protein kinase 2) belonging to the family of p38 MAPK $(144,146)$. Via SAPK2 signalling the actinpolymerization factor HSP27 is activated, thereby further increasing SF formation $(28,144)$ (Fig. 5). HSP90/RhoA-dependent phosphorylation of FAK is required for this Cdc42/SAPK2-mediated SF formation (144). The overexpression of Cdc42GAP indeed inhibited endothelial migration (147).

Interestingly, in tumor ECs lacking associated pericytes VEGFmediated Tyr951 phosphorylation of VEGFR2 binds TSAd (T-cellspecific adapter molecule) which is required for SF formation and motility (133), suggesting that this pathway is involved in RhoA activation. Although the cross-linking of RTK and GPCR signalling lies outside the scope of this review, we like to mention that VEGFinduced phosphorylation of VEGFR2 at Tyr951 also mediates activation of RhoA and Racl via the $G \alpha_{11 / Q}$ and $G_{\beta \gamma}$ subunits (19) ( Fig. 5). Additionally, VEGF-induced G-protein coupled PLC $\beta 3$ activation regulates migration of ECs and vascular sprouting by activation of Cdc42 (148).

$\mathrm{G} \alpha_{12 / 13}$ seems not to be involved in the cross-linking of RTK and GPCR signalling, which may explain why activated RhoA levels are much lower in VEGF-activated ECs than in thrombin-stimulated ECs (22). The different levels of RhoA activation may underlie functional differences between thrombin and VEGF signalling. Thrombin stimulates a generalised contraction of ECs, whereas VEGF-mediated migration involves retraction of the trailing edges of the cells, which nonetheless can lead to inter-endothelial gap formation (22).

\section{VEGF-induced Rho GEFs and GAPS}

Several Rho GTPase regulating proteins have been identified that are involved in VEGF-induced vascular responses in animal models. VEGF was shown to activate the RhoA-specific GEF ECT2 via the actin binding protein Kleip, a process that is further stimulated under hypoxia due to up-regulation of Kleip (149). Since Kleip/ ECT2 is required for cell migration (149), it seems reasonable to hypothesise that Kleip/ECT2 forms part of the HSP90-mediated 


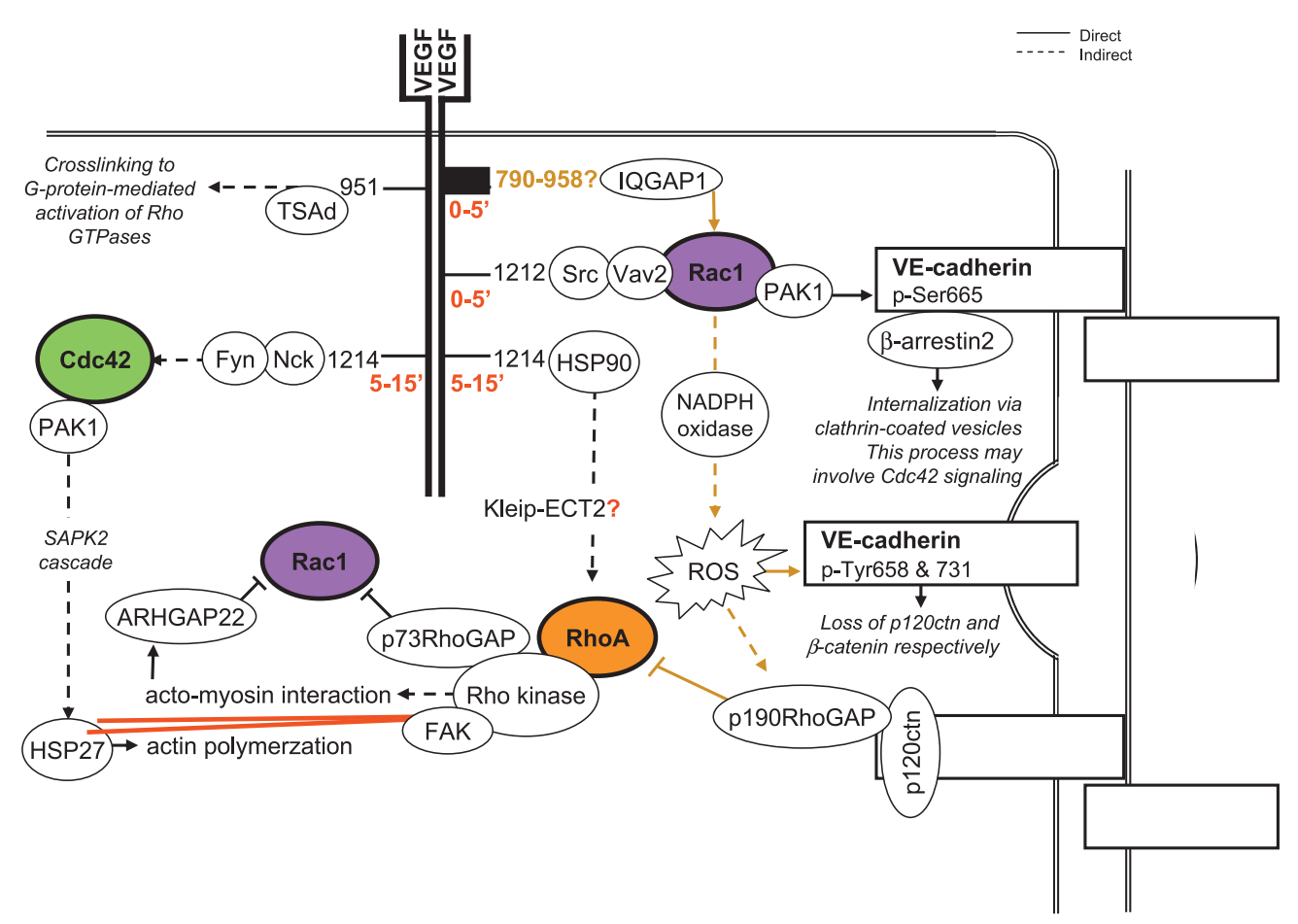

Figure 5: VEGF-mediated GTPase regulation induces endothelial permeability.VEGFR2 can be phosphorylated at multiple tyrosine sites of which three are indicated. Tyr1212 phosphorylation of VEGFR2 induces Rac1-mediated VE-cadherin internalisation and endothelial permeability. VEGF further induces Rac1-mediated ROS production, which via VE-cadherin phosphorylation at Tyr658 and Tyr731 induces the disassembly of p120ctn and

RhoA activation pathway (Fig. 5). In addition, the angiomotin/ RICH1 complex, which stabilises the TJs by maintaining Cdc42 activity (76), upon VEGF stimulation recruits the RhoGEF Syx, which mediates local RhoA activity $(150,151)$. Syx has been encountered in ECs in vivo $(150,151)$.

Rho kinase activity and the RhoA/Rho kinase-mediated actomyosin interaction cause activation of FILGAP ( 73 RhoGAP) and ARHGAP22, respectively. In HUVECs overexpression of p68RacGAP (the mouse equivalent of human ARHGAP22) indeed inhibited Racl-dependent lamellipodia formation and cell migration (152). Although not much is known about it, contractionmediated inhibition via p73RhoGAP and ARHGAP22 may explain the dip in theVEGF-mediated Racl activation after 5 min (see Fig. 1).

One may anticipate that these endothelial GAPs and GEFs also play a role in the regulation of VEGF-mediated permeability.

\section{Summing up}

VEGF/VEGFR2-mediated activation of Rac1 involves Vav2 and mediates VE-cadherin phosphorylation and internalisation. Meanwhile, Rac1 is also activated in a VEGFR2/IQGAP1-mediated manner, leading to ROS production. This results in tyrosine phos- $\beta$-catenin, respectively. ROS-mediated p190RhoGAP recruitment to the AJs locally inhibits RhoA. Rac1 thus dually mediates permeability and adhesion. Phosphorylation of Tyr1214 mediates RhoA and Cdc42 activation needed for acto-myosin interactions and SF formation. Finally, Tyr951 phosphorylation induces crosslinking to G-protein-mediated activation of Rho GTPases. It is likely that TSAd is involved in this process. For further detail see text.

phorylation of VE-cadherin, subsequent detachment of $\beta$-catenin and p120ctn, and increased permeability. ROS also induces a p190RhoGAP/p120ctn-mediated inactivation of RhoA. Cdc42 is further activated by VEGF-mediated inhibition of Cdc42GAP and VEGR2-phosphorylation. In the latter pathway PAK2 may be involved as a GEF for Cdc42. VEGF/VEGFR2/HSP90-mediated activation of RhoA occurs possibly via activation of Kleip and ECT2 and is involved in cell migration. In addition, RhoA activation by Syx occurs at the cell margins and is involved in protrusion formation. VEGF-mediated Rho/Rho kinase-activated p73RhoGAP, supported by the acto-myosin interaction-activated ARHGAP22, subsequently inhibits Rac1. And finally, VEGF-mediated activation of RhoA, Rac and Cdc42 was shown in a G-protein-mediated manner.

\section{Rho GTPases in permeability regulation by other agents}

Many other vascular mediators, such as platelet activating factor (153), hydrogen peroxide (154), oxidized LDL (155), bacterial toxins (156), TNF $\alpha$ (2), lysophosphatidic acid (LPA) (157), angiopoietins $(158,159)$ and also leukocytes can modulate Rho GTPase- 
mediated inter-endothelial gap formation. GTPase regulation by TNF $\alpha$ and LPA involves known but also new GEFs and GAPs.

Little is known about the Rho GTPase regulation by LPA in the endothelium. From other cell types it is known that, like thrombin, LPA mediates a FAK-dependent activation of RhoA via PDZ-Rho$\operatorname{GEF}(160,161)$. In addition, LPA enhances the expression of Net1, which positively effects RhoA activity and cell migration (162). The RhoA effector mDia was furthermore shown to associate with LARG thereby forming a positive feedback mechanism for LPAmediated RhoA activity (163). It is unknown whether these pathways also play a role in thrombin-mediated RhoA activation. The same is true for LPA-mediated activation of p116-Rho-interacting protein (p116RIP), a RhoA specific GAP (164) that simultaneously binds RhoA and MYPT1, the regulatory subunit of MLCP. This way LPA activates MLCP and simultaneously inhibits RhoA, thus dually suppressing acto-myosin interaction and cell contraction (165).

Like thrombin, TNF $\alpha$ induces RhoA-mediated SF formation, cell contraction and endothelial permeability (2). Unlike thrombin, TNF-induced GEF-H1-mediated RhoA activation, was shown downstream of TNF $\alpha$-induced ERK signalling (166). Furthermore, TNF $\alpha$ induces the expression of interleukin (IL)-6 and IL-8. This involves TNF $\alpha$-mediated Rac1 activation, which modulates p68, a subunit of the transcription factor NF- $\mathrm{KB}(167,168)$. To date however, it is not known how this Racl activity is regulated. TNF $\alpha$ signalling involves factor-associated-with-nSMase (FAN), an adaptor protein that links the plasma membrane to the actin cytoskeleton. FAN is a key mediator of TNF $\alpha$-induced Cdc42 activation and filopodia formation (169). The RhoGEF that links FAN to Cdc42 activation has not yet been identified.

Angiopoietins recently have drawn a lot of attention as modifiers of endothelial barrier function through modulation of Rho GTPase activity $(158,159)$, as was reviewed by van der Heijden et al. (159). Angiopoietins are growth factors known to function as ligands for the RTK Tie2. Angiopoietin (Ang)-2 provokes inflammation and vascular hyperpermeability, while Ang-1 has a protective effect. The ratio of the circulating levels of Ang-1 and Ang-2 strongly associates with pulmonary microvascular permeability in diseases such as ARDS (159). Ang-2 inhibits phosphorylation of the Tie 2 receptor, which increased RhoA and Rho-kinase activity (158). In contrast, Ang-1-induced Tie2 receptor phosphorylation, which signals via PI3-kinase and Rac1 to activate p190RhoGAP (159). Alternatively, Ang-1 might act by promoting the activation of $\mathrm{mDia}$ through RhoA, resulting in the association of $\mathrm{mDia}$ with Src. This deprives VEGFRs of an essential molecule required for the disruption of endothelial cell-cell contacts and paracellular permeability (14). This barrier-protective effect of RhoA through $\mathrm{mDia}$ is in apparent contrast to the response of e.g. thrombin and LPS, which enhance vascular permeability through a robust and persistent RhoA activation and the consequent Rho kinase-dependent assembly of SFs and cell contraction. The strength and location of RhoA activation may therefore affect the choice of the RhoA downstream target, thereby determining whether RhoAinitiated signals protect the barrier function of ECs or promote vascular leakage.
Finally, leukocyte activation of the ECs induces clustering of ICAM-1. This stimulates the Rho specific SGEF, which activates the small GTPase RhoG (170). The dorsal ruffles that are subsequently formed assemble a docking structure around the leukocyte and enable leukocyte migration throught the EC monolayer. In addition to SGEF, the Rho/Rac GEF Trio may also be activated downstream of ICAM-1 clustering, since it associates to filamin.

\section{Conclusion and perspectives}

Rho GTPases, which were discovered as regulatory proteins that regulate specific actin cytoskeletal structures - SF by RhoA, lamellipodia by Rac1 and filopodia by Cdc42 - are now known as complex integrators of cytoskeletal structures that can have multiple functions depending on the cellular context. Fundamental to these functions are three-dimensional complexes between the individual Rho GTPases, their specific GEFs and GAPs, which largely outnumber the Rho GTPases themselves, and additional regulatory proteins. By this complexity of regulation different vasoactive mediators can induce various cytoskeletal structures that enable the ECs to respond adequately. In this review we have focused on the consequences of Rho GTPase regulation for endothelial barrier function.

From the foregoing discussion it has become clear that GPCRand RTK-mediated activations of Rho GTPases have markedly different effects. The G-protein coupled thrombin receptor PAR-1 induces mainly RhoA-mediated cell rounding, whereas RTK VEGFR2, by means of VEGF, induces protrusion, cell migration and angiogenesis. Thus the initial idea to investigate Rho GTPase signalling in order to find a therapeutic target to counteract permeability seems logical. However, we have seen here that many different and even independent pathways lead to thrombin-mediated activation of RhoA. Moreover, there are several pathways that independently result in the stimulation of different Rho GEFs and GAPs, suggesting that targeting just one of them is not going to efficiently inhibit RhoA activation and permeability.

In contrast, VEGF-mediated Rho GTPase regulation shows that the fine-tuning of RhoA, Racl and Cdc42 activities drives different processes at the same time. The spatio-temporal regulation is crucial here and may thus form an interesting opportunity to interfere with one process while stimulating another.

An interesting feature of Rho GTPases in endothelial barrier regulation is that they often have both barrier protecting and barrier disturbing functions. While Racl in most conditions enforces the junctions that form the endothelial barrier, it becomes part of a barrier disturbing mechanism as activator of NADPH oxidase, which generates ROS. Similarly RhoA has a dual role in barrier regulation, being protective in basal conditions, but strongly involved in barrier dysfunction after activation of ECs by thrombin.

At present most information on Rho GTPases has been acquired from studies in cultured cells. Cultured ECs display certain characteristics more pronounced than their in vivo counterparts, e.g. the extent of SF formation, while other aspects are more domi- 
nant in vivo. Future studies have to clarify the importance of various parts of the signalling pathways in pathological conditions in vivo. Both studies in transgenic animals and evaluation of clinical samples are needed to assemble the insight needed for pharmacological intervention in man. In this context, location-specific GTPase regulators, preferably EC-specific GEFs and GAPs, may be the most interesting to modulate the formation of cell protrusions and migration or endothelial permeability. The challenge today is to unfold their regulation, thereby not only looking at the GTPase for which they have the highest GEF or GAP affinity, but also determining their effect on the other GTPases since it is this cross-talk, or the lack of it, that makes them an interesting therapeutic target.

\section{References}

1. Aepfelbacher M, Essler M, Huber E, et al. Bacterial toxins block endothelial wound repair. Evidence that Rho GTPases control cytoskeletal rearrangements in migrating endothelial cells. Arterioscler Thromb Vasc Biol 1997; 17: 1623-1629.

2. Wojciak-Stothard B, Entwistle A, Garg R, et al. Regulation of TNF-alpha-induced reorganization of the actin cytoskeleton and cell-cell junctions by Rho, Rac, and Cdc42 in human endothelial cells. J Cell Physiol 1998; 176: 150-165.

3. Uehata M, Ishizaki $\mathrm{T}$, Satoh $\mathrm{H}$, et al. Calcium sensitization of smooth muscle mediated by a Rho-associated protein kinase in hypertension. Nature 1997; 389: 990-994.

4. van Nieuw Amerongen GP, van Hinsbergh VW. Cytoskeletal effects of rho-like small guanine nucleotide-binding proteins in the vascular system. Arterioscler Thromb Vasc Biol 2001; 21: 300-311.

5. Etienne-Manneville S, Hall A. Rho GTPases in cell biology. Nature 2002; 420: 629-635.

6. De Smet F, Segura I, De Bock K, et al. Mechanisms of vessel branching: filopodia on endothelial tip cells lead the way. Arterioscler Thromb Vasc Biol 2009; 29: 639-649.

7. Ridley AJ. Rho family proteins: coordinating cell responses. Trends Cell Biol 2001; 11: 471-477.

8. Aspenstrom P, Ruusala A, Pacholsky D. Taking Rho GTPases to the next level: the cellular functions of atypical Rho GTPases. Exp Cell Res 2007; 313: 3673-3679.

9. Aghajanian A, Wittchen ES, Allingham MJ, et al. Endothelial cell junctions and the regulation of vascular permeability and leukocyte transmigration. J Thromb Haemost 2008; 6: 1453-1460.

10. Lampugnani MG, Zanetti A, Breviario F, et al. VE-cadherin regulates endothelia actin activating Rac and increasing membrane association of Tiam. Mol Biol Cell 2002; 13: 1175-1189.

11. Vandenbroucke E, Mehta D, Minshall R, et al. Regulation of endothelial junctional permeability. Ann NY Acad Sci 2008; 1123: 134-145.

12. Wojciak-Stothard B, Ridley AJ. Rho GTPases and the regulation of endothelial permeability. Vascul Pharmacol 2002; 39: 187-199.

13. van Nieuw Amerongen GP, Beckers CM, Achekar ID, et al. Involvement of Rho kinase in endothelial barrier maintenance. Arterioscler Thromb Vasc Biol 2007; 27: 2332-2339.

14. Gavard J, Patel V, Gutkind JS. Angiopoietin-1 prevents VEGF-induced endothelial permeability by sequestering Src through mDia. Dev Cell 2008; 14: 25-36.

15. Baumer Y, Drenckhahn D, Waschke J. cAMP induced Rac 1-mediated cytoskeletal reorganization in microvascular endothelium. Histochem Cell Biol 2008; 129: 765-778.

16. Garcia JG, Liu F, Verin AD, et al. Sphingosine 1-phosphate promotes endothelial cell barrier integrity by Edg-dependent cytoskeletal rearrangement. J Clin Invest 2001; 108: 689-701.

17. Tauseef M, Kini V, Knezevic N, et al D. Activation of sphingosine kinase-1 reverses the increase in lung vascular permeability through sphingosine-1-phosphate receptor signalling in endothelial cells. Circ Res 2008; 103: 1164-1172.

18. Temmesfeld-Wollbruck B, Hocke AC, Suttorp N, et al. Adrenomedullin and endothelial barrier function. Thromb Haemost 2007; 98: 944-951.

19. Zeng H, Zhao D, Mukhopadhyay D. KDR stimulates endothelial cell migration through heterotrimeric $\mathrm{G}$ protein $\mathrm{Gq} / 11$-mediated activation of a small GTPase RhoA. J Biol Chem 2002; 277: 46791-46798.
20. Broman MT, Mehta D, Malik AB. Cdc42 regulates the restoration of endothelial adherens junctions and permeability. Trends Cardiovasc Med 2007; 17: 151-156.

21. van Nieuw Amerongen GP, van Delft S, Vermeer MA, et al. Activation of RhoA by thrombin in endothelial hyperpermeability: role of Rho kinase and protein tyrosine kinases. Circ Res 2000; 87: 335-340.

22. van Nieuw Amerongen GP, Koolwijk P, Versteilen A, et al. Involvement of RhoA/ Rho kinase signalling in VEGF-induced endothelial cell migration and angiogenesis in vitro. Arterioscler Thromb Vasc Biol 2003; 23: 211-217.

23. Garrett TA, Van Buul JD, Burridge K. VEGF-induced Racl activation in endothelial cells is regulated by the guanine nucleotide exchange factor Vav2. Exp Cell Res 2007; 313: 3285-3297.

24. Rosenfeldt H, Castellone MD, Randazzo PA, et al. Rac inhibits thrombin-induced Rho activation: evidence of a Pak-dependent GTPase crosstalk. J Mol Signal 2006; $1: 8$.

25. Mehta D, Malik AB. Signalling mechanisms regulating endothelial permeability. Physiol Rev 2006; 86: 279-367.

26. Monaghan-Benson E, Burridge K. The regulation of VEGF-induced microvascular permeability requires Rac and ROS. J Biol Chem 2009; 284: 25602-25611..

27. Nimnual AS, Taylor LJ, Bar-Sagi D. Redox-dependent downregulation of Rho by Rac. Nat Cell Biol 2003; 5: 236-241.

28. Rousseau S, Houle F, Huot J. Integrating the VEGF signals leading to actin-based motility in vascular endothelial cells. Trends Cardiovasc Med 2000; 10:321-327.

29. Siderovski DP, Willard FS. The GAPs, GEFs, and GDIs of heterotrimeric G-protein alpha subunits. Int J Biol Sci 2005; 1: 51-66.

30. Rossman KL, Der CJ, Sondek J. GEF means go: turning on RHO GTPases with guanine nucleotide-exchange factors. Nat Rev Mol Cell Biol 2005; 6: 167-180.

31. Erickson JW, Cerione RA. Structural elements, mechanism, and evolutionary convergence of Rho protein-guanine nucleotide exchange factor complexes. Biochemistry 2004; 43: 837-842.

32. Cerione RA, Zheng Y. The Dbl family of oncogenes. Curr Opin Cell Biol 1996; 8: 216-222.

33. Schmidt A, Hall A. Guanine nucleotide exchange factors for Rho GTPases: turning on the switch. Genes Dev 2002; 16: 1587-1609.

34. Hart MJ, Jiang X, Kozasa T, et al. Direct stimulation of the guanine nucleotide exchange activity of p115 RhoGEF by Galpha13. Science 1998; 280: 2112-2114.

35. Kozasa T, Jiang X, Hart MJ, et al. p115 RhoGEF, a GTPase activating protein for Galpha12 and Galpha13. Science 1998; 280: 2109-2111.

36. Longenecker KL, Lewis ME, Chikumi H, et al. Structure of the RGS-like domain from PDZ-RhoGEF: linking heterotrimeric g protein-coupled signalling to Rho GTPases. Structure 2001; 9: 559-569.

37. Sanz-Moreno V, Gadea G, Ahn J, et al. Rac activation and inactivation control plasticity of tumor cell movement. Cell 2008; 135: 510-523.

38. Lents NH, Irintcheva V, Goel R, et al. The rapid activation of N-Ras by alphathrombin in fibroblasts is mediated by the specific G-protein Galpha(i2)Gbeta(1)-Ggamma(5) and occurs in lipid rafts. Cell Signal 2009; 21: 1007-1014.

39. Cote JF, Vuori K. Identification of an evolutionarily conserved superfamily of DOCK180-related proteins with guanine nucleotide exchange activity. J Cell Sci 2002; 115: 4901-4913.

40. Cote JF, Vuori K. GEF what? Dock180 and related proteins help Rac to polarize cells in new ways. Trends Cell Biol 2007; 17: 383-393.

41. Fukui Y, Hashimoto O, Sanui T, et al. Haematopoietic cell-specific CDM family protein DOCK2 is essential for lymphocyte migration. Nature 2001; 412: 826-831.

42. Siehler S. Regulation of RhoGEF proteins by G(12/13)-coupled receptors. Br J Pharmacol 2009; 158: 41-49.

43. Chikumi H, Fukuhara S, Gutkind JS. Regulation of G protein-linked guanine nucleotide exchange factors for Rho, PDZ-RhoGEF, and LARG by tyrosine phosphorylation: evidence of a role for focal adhesion kinase. J Biol Chem 2002; 277: 12463-12473.

44. Schiller MR. Coupling receptor tyrosine kinases to Rho GTPases--GEFs what's the link. Cell Signal 2006; 18: 1834-1843.

45. Schmidt A, Hall A. The Rho exchange factor Net1 is regulated by nuclear sequestration. J Biol Chem 2002; 277: 14581-14588.

46. Tatsumoto T, Xie X, Blumenthal R, et al. Human ECT2 is an exchange factor for Rho GTPases, phosphorylated in G2/M phases, and involved in cytokinesis. J Cell Biol 1999; 147: 921-928.

47. Michiels F, Stam JC, Hordijk PL, et al. Regulated membrane localization of Tiam1, mediated by the NH2-terminal pleckstrin homology domain, is required for Racdependent membrane ruffling and C-Jun NH2-terminal kinase activation. J Cell Biol 1997; 137: 387-398. 
48. Krendel M, Zenke FT, Bokoch GM. Nucleotide exchange factor GEF-H1 mediates cross-talk between microtubules and the actin cytoskeleton. Nat Cell Biol 2002; 4 : 294-301.

49. Birukova AA, Adyshev D, Gorshkov B, et al. GEF-H1 is involved in agonist-induced human pulmonary endothelial barrier dysfunction. Am J Physiol Lung Cell Mol Physiol 2006; 290: L540-L548.

50. Callow MG, Zozulya S, Gishizky ML, et al. PAK4 mediates morphological changes through the regulation of GEF-H1. J Cell Sci 2005; 118: 1861-1872.

51. Ohta Y, Hartwig JH, Stossel TP. FilGAP, a Rho- and ROCK-regulated GAP for Rac binds filamin A to control actin remodelling. Nat Cell Biol 2006; 8: 803-814.

52. Su ZJ, Hahn CN, Goodall GJ, et al. A vascular cell-restricted RhoGAP, p73RhoGAP, is a key regulator of angiogenesis. Proc Natl Acad Sci USA 2004; 101: 12212-12217.

53. Tcherkezian J, Lamarche-Vane N. Current knowledge of the large RhoGAP family of proteins. Biol Cell 2007; 99: 67-86.

54. Buchsbaum RJ. Rho activation at a glance. J Cell Sci 2007; 120: 1149-1152.

55. Dovas A, Couchman JR. RhoGDI: multiple functions in the regulation of Rho family GTPase activities. Biochem J 2005; 390: 1-9.

56. Olofsson B. Rho guanine dissociation inhibitors: pivotal molecules in cellular signalling. Cell Signal 1999; 11: 545-554.

57. Fu P, Birukov KG. Oxidized phospholipids in control of inflammation and endothelial barrier. Transl Res 2009; 153: 166-176.

58. Wildenberg GA, Dohn MR, Carnahan RH, et al. p120-catenin and p190RhoGAP regulate cell-cell adhesion by coordinating antagonism between Rac and Rho. Cell 2006; 127: 1027-1039.

59. Braga VM, Machesky LM, Hall A, et al. The small GTPases Rho and Rac are required for the establishment of cadherin-dependent cell-cell contacts. J Cell Biol 1997; 137: 1421-1431.

60. Noren NK, Niessen CM, Gumbiner BM, et al. Cadherin engagement regulates Rho family GTPases. J Biol Chem 2001; 276: 33305-33308.

61. Yamada S, Nelson WJ. Localized zones of Rho and Rac activities drive initiation and expansion of epithelial cell-cell adhesion. J Cell Biol 2007; 178: 517-527.

62. Tan W, Palmby TR, Gavard J, et al. An essential role for Rac1 in endothelial cell function and vascular development. FASEB J 2008; 22: 1829-1838.

63. Patterson C. Torturing a blood vessel. Nat Med 2009; 15: 137-138.

64. Kleaveland B, Zheng X, Liu JJ, et al. Regulation of cardiovascular development and integrity by the heart of glass-cerebral cavernous malformation protein pathway. Nat Med 2009; 15: 169-176.

65. Whitehead KJ, Chan AC, Navankasattusas S, et al. The cerebral cavernous malformation signalling pathway promotes vascular integrity via Rho GTPases. Nat Med 2009; 15: 177-184.

66. Crose LE, Hilder TL, Sciaky N, et al. Cerebral cavernous malformation 2 protein promotes smad ubiquitin regulatory factor 1-mediated RhoA degradation in endothelial cells. J Biol Chem 2009; 284: 13301-13305.

67. Ozdamar B, Bose R, Barrios-Rodiles M, et al. Regulation of the polarity protein Par6 by TGFbeta receptors controls epithelial cell plasticity. Science 2005; 307: 1603-1609.

68. Chauvet N, Prieto M, Fabre C, et al. Distribution of p120 catenin during rat brain development: potential role in regulation of cadherin-mediated adhesion and actin cytoskeleton organization. Mol Cell Neurosci 2003; 22: 467-486.

69. Noren NK, Liu BP, Burridge K, et al. p120 catenin regulates the actin cytoskeleton via Rho family GTPases. J Cell Biol 2000; 150: 567-580.

70. Brill S, Li S, Lyman CW, et al. The Ras GTPase-activating-protein-related human protein IQGAP2 harbors a potential actin binding domain and interacts with calmodulin and Rho family GTPases. Mol Cell Biol 1996; 16: 4869-4878.

71. Ho YD, Joyal JL, Li Z, et al. IQGAP1 integrates Ca2+/calmodulin and Cdc42 signalling. J Biol Chem 1999; 274: 464-470.

72. Hart MJ, Callow MG, Souza B, et al. IQGAP1, a calmodulin-binding protein with a rasGAP-related domain, is a potential effector for cdc42Hs. EMBO J 1996; 15 : 2997-3005.

73. Bashour AM, Fullerton AT, Hart MJ, et al. IQGAP1, a Rac- and Cdc42-binding protein, directly binds and cross-links microfilaments. J Cell Biol 1997; 137: 1555-1566.

74. Fukata M, Nakagawa M, Itoh N, et al. Involvement of IQGAP1, an effector of Racl and Cdc42 GTPases, in cell-cell dissociation during cell scattering. Mol Cell Biol 2001; 21: 2165-2183.

75. Fukata M, Nakagawa M, Kuroda S, et al. Cell adhesion and Rho small GTPases. J Cell Sci 1999; 112: 4491-4500.

76. Wells CD, Fawcett JP, Traweger A, et al. A Rich1/Amot complex regulates the Cdc42 GTPase and apical-polarity proteins in epithelial cells. Cell 2006; 125: 535-548.
77. Braga VM. Cell-cell adhesion and signalling. Curr Opin Cell Biol 2002; 14: 546-556.

78. Reynolds AB, Herbert L, Cleveland JL, et al. p120, a novel substrate of protein tyrosine kinase receptors and of p60v-src, is related to cadherin-binding factors beta-catenin, plakoglobin and armadillo. Oncogene 1992; 7: 2439-2445.

79. Sousa S, Cabanes D, Archambaud C, et al. ARHGAP10 is necessary for alpha-catenin recruitment at adherens junctions and for Listeria invasion. Nat Cell Biol 2005; 7: 954-960.

80. Birukova AA, Zagranichnaya T, Alekseeva E, et al. Epac/Rap and PKA are novel mechanisms of ANP-induced Rac-mediated pulmonary endothelial barrier protection. J Cell Physiol 2008; 215: 715-724.

81. Herbrand U, Ahmadian MR. p190-RhoGAP as an integral component of the Tiam1/Racl-induced downregulation of Rho. Biol Chem 2006; 387: 311-317.

82. Qiao J, Holian O, Lee BS, et al. Phosphorylation of GTP dissociation inhibitor by PKA negatively regulates RhoA. Am J Physiol Cell Physiol 2008; 295: C1161-C1168.

83. Sakurai A, Fukuhara S, Yamagishi A, et al. MAGI-1 is required for Rap1 activation upon cell-cell contact and for enhancement of vascular endothelial cadherin-mediated cell adhesion. Mol Biol Cell 2006; 17: 966-976.

84. Asuri S, Yan J, Paranavitana NC, et al. E-cadherin dis-engagement activates the Rap1 GTPase. J Cell Biochem 2008; 105: 1027-1037.

85. Kobielak A, Fuchs E. Alpha-catenin: at the junction of intercellular adhesion and actin dynamics. Nat Rev Mol Cell Biol 2004; 5: 614-625.

86. Wong KW, Mohammadi S, Isberg RR. Disruption of RhoGDI and RhoA regulation by a Racl specificity switch mutant. J Biol Chem 2006; 281:40379-40388.

87. Klarenbach SW, Chipiuk A, Nelson RC, et al. Differential actions of PAR2 and PAR1 in stimulating human endothelial cell exocytosis and permeability: the role of Rho-GTPases. Circ Res 2003; 92: 272-278.

88. McLaughlin JN, Shen L, Holinstat M, et al. Functional selectivity of G protein signalling by agonist peptides and thrombin for the protease-activated receptor-1. J Biol Chem 2005; 280: 25048-25059.

89. Vogt S, Grosse R, Schultz G, et al. Receptor-dependent RhoA activation in G12/G13-deficient cells: genetic evidence for an involvement of Gq/G11. J Biol Chem 2003; 278: 28743-28749.

90. Hung DT, Wong YH, Vu TK, et al. The cloned platelet thrombin receptor couples to at least two distinct effectors to stimulate phosphoinositide hydrolysis and inhibit adenylyl cyclase. J Biol Chem 1992; 267: 20831-20834.

91. Holinstat M, Mehta D, Kozasa T, et al. Protein kinase Calpha-induced p115RhoGEF phosphorylation signals endothelial cytoskeletal rearrangement. J Biol Chem 2003; 278: 28793-28798.

92. Gavard J, Gutkind JS. Protein kinase C-related kinase and ROCK are required for thrombin-induced endothelial cell permeability downstream from Galpha12/13 and Galpha11/q. J Biol Chem 2008; 283: 29888-29896.

93. Kouklis P, Konstantoulaki M, Vogel S, et al. Cdc42 regulates the restoration of endothelial barrier function. Circ Res 2004; 94: 159-166.

94. Garcia JG, Lazar V, Gilbert-McClain LI, et al. Myosin light chain kinase in endothelium: molecular cloning and regulation. Am J Respir Cell Mol Biol 1997; 16: 489-494.

95. Goeckeler ZM, Wysolmerski RB. Myosin phosphatase and cofilin mediate cAMP/ cAMP-dependent protein kinase-induced decline in endothelial cell isometric tension and myosin II regulatory light chain phosphorylation. J Biol Chem 2005; 280: 33083-33095.

96. Kimura K, Ito M, Amano M, et al. Regulation of myosin phosphatase by Rho and Rho-associated kinase (Rho-kinase). Science 1996; 273: 245-248.

97. Satpathy M, Gallagher P, Lizotte-Waniewski M, et al. Thrombin-induced phosphorylation of the regulatory light chain of myosin II in cultured bovine corneal endothelial cells. Exp Eye Res 2004; 79: 477-486.

98. van Nieuw Amerongen GP, Draijer R, Vermeer MA, et al. Transient and prolonged increase in endothelial permeability induced by histamine and thrombin: role of protein kinases, calcium, and RhoA. Circ Res 1998; 83: 1115-1123.

99. Singh I, Knezevic N, Ahmmed GU, et al. Galphaq-TRPC6-mediated Ca2+ entry induces RhoA activation and resultant endothelial cell shape change in response to thrombin. J Biol Chem 2007; 282: 7833-7843.

100. Knezevic N, Roy A, Timblin B, et al. GDI-1 phosphorylation switch at serine 96 induces RhoA activation and increased endothelial permeability. Mol Cell Biol 2007; 27: 6323-6333.

101. Mehta D, Rahman A, Malik AB. Protein kinase C-alpha signals rho-guanine nucleotide dissociation inhibitor phosphorylation and rho activation and regulates the endothelial cell barrier function. J Biol Chem 2001; 276: 22614-22620.

102. Birukova AA, Smurova K, Birukov KG, et al. Role of Rho GTPases in thrombin- 
induced lung vascular endothelial cells barrier dysfunction. Microvasc Res 2004; 67: 64-77.

103. Kawano Y, Fukata Y, Oshiro N, et al. Phosphorylation of myosin-binding subunit (MBS) of myosin phosphatase by Rho-kinase in vivo. J Cell Biol 1999; 147 1023-1038.

104. Essler M, Amano M, Kruse HJ, et al. Thrombin inactivates myosin light chain phosphatase via Rho and its target Rho kinase in human endothelial cells. J Biol Chem 1998; 273: 21867-21874.

105. Amano M, Ito M, Kimura K, et al. Phosphorylation and activation of myosin by Rho-associated kinase (Rho-kinase). J Biol Chem 1996; 271: 20246-20249.

106. Riento K, Ridley AJ. Rocks: multifunctional kinases in cell behaviour. Nat Rev Mol Cell Biol 2003; 4: 446-456.

107. Mori K, Amano M, Takefuji M, et al. Rho-kinase contributes to sustained RhoA activation through phosphorylation of p190A RhoGAP. J Biol Chem 2008; 284: 5067-5076.

108. Holinstat M, Knezevic N, Broman M, et al. Suppression of RhoA activity by focal adhesion kinase-induced activation of p190RhoGAP: role in regulation of endothelial permeability. J Biol Chem 2006; 281: 2296-2305.

109. Settleman J, Albright CF, Foster LC, et al. Association between GTPase activators for Rho and Ras families. Nature 1992; 359: 153-154.

110. Ridley AJ, Self AJ, Kasmi F, et al. rho family GTPase activating proteins p190, bcr and rhoGAP show distinct specificities in vitro and in vivo. EMBO J 1993; 12 5151-5160.

111. Suzuki N, Hajicek N, Kozasa T. Regulation and physiological functions of G12/13-mediated signalling pathways. Neurosignals 2009; 17: 55-70.

112. Kanthou C, Kanse SM, Kakkar VV, et al. Involvement of pertussis toxin-sensitive and -insensitive $\mathrm{G}$ proteins in alpha-thrombin signalling on cultured human vascular smooth muscle cells. Cell Signal 1996; 8: 59-66.

113. Westendorp RG, Draijer R, Meinders AE, et al. Cyclic-GMP-mediated decrease in permeability of human umbilical and pulmonary artery endothelial cell monolayers. J Vasc Res 1994; 31: 42-51.

114. Heller R, Chang Q, Ehrlich G, et al. Overlapping and distinct roles for PI3Kbeta and gamma isoforms in S1P-induced migration of human and mouse endothelial cells. Cardiovasc Res 2008; 80: 96-105.

115. Diebold I, Djordjevic T, Petry A, et al. Phosphodiesterase 2 Mediates Redox-Sensitive Endothelial Cell Proliferation and Angiogenesis by Thrombin via Rac1 and NADPH Oxidase 2. Circ Res 2009; 104: 1169-1177.

116. Vouret-Craviari V, Bourcier C, Boulter E, et al. Distinct signals via Rho GTPases and Src drive shape changes by thrombin and sphingosine-1-phosphate in endothelial cells. J Cell Sci 2002; 115: 2475-2484.

117. Wojciak-Stothard B, Potempa S, Eichholtz T, et al. Rho and Rac but not Cdc42 regulate endothelial cell permeability. J Cell Sci 2001; 114: 1343-1355.

118. Luna A, Matas OB, Martinez-Menarguez JA, et al. Regulation of protein transport from the Golgi complex to the endoplasmic reticulum by CDC42 and N-WASP. Mol Biol Cell 2002; 13: 866-879.

119. Erickson JW, Zhang C, Kahn RA, et al. Mammalian Cdc42 is a brefeldin A-sensitive component of the Golgi apparatus. J Biol Chem 1996; 271: 26850-26854.

120. Nakamura T, Hayashi T, Nasu-Nishimura Y, et al. PX-RICS mediates ER-toGolgi transport of the N-cadherin/beta-catenin complex. Genes Dev 2008; 22 1244-1256.

121. Wang B, Wylie FG, Teasdale RD, et al. Polarized trafficking of E-cadherin is regulated by Racl and Cdc42 in Madin-Darby canine kidney cells. Am J Physiol Cell Physiol 2005; 288: C1411-C1419.

122. Vogel SM, Gao X, Mehta D, et al. Abrogation of thrombin-induced increase in pulmonary microvascular permeability in PAR-1 knockout mice. Physiol Genomics 2000; 4: 137-145.

123. Garcia JG, Pavalko FM, Patterson CE. Vascular endothelial cell activation and permeability responses to thrombin. Blood Coagul Fibrinolysis 1995; 6: 609-626.

124. Andriopoulou P, Navarro P, Zanetti A, et al. Histamine induces tyrosine phosphorylation of endothelial cell-to-cell adherens junctions. Arterioscler Thromb Vasc Biol 1999; 19: 2286-2297.

125. McLachlan RW, Yap AS. Not so simple: the complexity of phosphotyrosine signalling at cadherin adhesive contacts. J Mol Med 2007; 85: 545-554.

126. Majno G, Gilmore V, Leventhal M. On the mechanism of vascular leakage caused by histaminetype mediators. A microscopic study in vivo. Circ Res 1967;21:833-847.

127. van Nieuw Amerongen GP, Musters RJ, Eringa EC, et al. Thrombin-induced en dothelial barrier disruption in intact microvessels: role of RhoA/Rho kinasemyosin phosphatase axis. Am J Physiol Cell Physiol 2008; 294: C1234-C1241.
128. Baluk P, Hirata A, Thurston G, et al. Endothelial gaps: time course of formation and closure in inflamed venules of rats. Am J Physiol 1997; 272: L155-L170.

129. Kaneider NC, Leger AJ, Agarwal A, et al. 'Role reversal' for the receptor PAR1 in sepsis-induced vascular damage. Nat Immunol 2007; 8: 1303-1312.

130. Schuepbach RA, Feistritzer C, Fernandez JA, et al. Protection of vascular barrier integrity by activated protein $\mathrm{C}$ in murine models depends on protease-activated receptor-1. Thromb Haemost 2009; 101: 724-733.

131. Bae JS, Rezaie AR. Protease activated receptor 1 (PAR-1) activation by thrombin is protective in human pulmonary artery endothelial cells if endothelial protein $\mathrm{C}$ receptor is occupied by its natural ligand. Thromb Haemost 2008; 100: 101-109.

132. Hofer E, Schweighofer B. Signal transduction induced in endothelial cells by growth factor receptors involved in angiogenesis. Thromb Haemost 2007; 97: 355-363.

133. Olsson AK, Dimberg A, Kreuger J, et al. VEGF receptor signalling - in control of vascular function. Nat Rev Mol Cell Biol 2006; 7: 359-371.

134. Carmeliet P, Lampugnani MG, Moons L, et al. Targeted deficiency or cytosolic truncation of the VE-cadherin gene in mice impairs VEGF-mediated endothelial survival and angiogenesis. Cell 1999; 98: 147-157.

135. Yamaoka-Tojo M, Tojo T, Kim HW, et al. IQGAP1 mediates VE-cadherin-based cell-cell contacts and VEGF signalling at adherence junctions linked to angiogenesis. Arterioscler Thromb Vasc Biol 2006; 26: 1991-1997.

136. Weis S, Cui J, Barnes L, et al. Endothelial barrier disruption by VEGF-mediated Src activity potentiates tumor cell extravasation and metastasis. J Cell Biol 2004; 167: 223-229.

137. Soldi R, Mitola S, Strasly M, et al. Role of alphavbeta3 integrin in the activation of vascular endothelial growth factor receptor-2. EMBO J 1999; 18: 882-892.

138. Takahashi T, Yamaguchi S, Chida K, et al. A single autophosphorylation site on KDR/Flk-1 is essential for VEGF-A-dependent activation of PLC-gamma and DNA synthesis in vascular endothelial cells. EMBO J 2001; 20: 2768-2778.

139. Meyer RD, Dayanir V, Majnoun F, et al. The presence of a single tyrosine residue at the carboxyl domain of vascular endothelial growth factor receptor-2/FLK-1 regulates its autophosphorylation and activation of signalling molecules. J Biol Chem 2002; 277: 27081-27087.

140. Eriksson A, Cao R, Roy J, et al. Small GTP-binding protein Rac is an essential mediator of vascular endothelial growth factor-induced endothelial fenestrations and vascular permeability. Circulation 2003; 107: 1532-1538.

141. Gavard J, Gutkind JS.VEGF controls endothelial-cell permeability by promoting the beta-arrestin-dependent endocytosis of VE-cadherin. Nat Cell Biol 2006; 8: 1223-1234.

142. Baumeister U, Funke R, Ebnet K, et al. Association of Csk to VE-cadherin and inhibition of cell proliferation. EMBO J 2005; 24: 1686-1695.

143. Yamaoka-Tojo M, Ushio-Fukai M, Hilenski L, et al. IQGAP1, a novel vascular endothelial growth factor receptor binding protein, is involved in reactive oxygen species--dependent endothelial migration and proliferation. Circ Res 2004; 95 : 276-283.

144. Lamalice L, Le Boeuf F, Huot J. Endothelial cell migration during angiogenesis. Circ Res 2007; 100: 782-794.

145. Holmqvist K, Cross MJ, Rolny C, et al. The adaptor protein shb binds to tyrosine 1175 in vascular endothelial growth factor (VEGF) receptor-2 and regulates VEGF-dependent cellular migration. J Biol Chem 2004; 279: 22267-22275.

146. Zhang S, Han J, Sells MA, et al. Rho family GTPases regulate p38 mitogen-activated protein kinase through the downstream mediator Pak1. J Biol Chem 1995; 270: 23934-23936.

147. Engelse MA, Laurens N, Verloop RE, et al. Differential gene expression analysis of tubule forming and non-tubule forming endothelial cells: CDC42GAP as a counter-regulator in tubule formation. Angiogenesis 2008; 11: 153-167.

148. Bhattacharya R, Kwon J, Li X, et al. Distinct role of PLC $\{$ beta\} 3 in VEGF-mediated directional migration and vascular sprouting. J Cell Sci 2009; 122: 1025-1034.

149. Nacak TG, Alajati A, Leptien K, et al. The BTB-Kelch protein KLEIP controls endothelial migration and sprouting angiogenesis. Circ Res 2007; 100: 1155-1163.

150. Ernkvist M, Persson NL, Audebert S, et al. The Amot/Patj/Syx signalling complex spatially controls RhoA GTPase activity in migrating endothelial cells. Blood 2009; 113: 244-253.

151. Garnaas MK, Moodie KL, Liu ML, et al. Syx, a RhoA guanine exchange factor, is essential for angiogenesis in vivo. Circ Res 2008; 103: 710-716.

152. Aitsebaomo J, Wennerberg K, Der CJ, et al. p68RacGAP is a novel GTPase-acti- 
vating protein that interacts with vascular endothelial zinc finger-1 and modulates endothelial cell capillary formation. J Biol Chem 2004; 279: 17963-17972.

153. Knezevic II, Predescu SA, Neamu RF, et al. Tiaml and Racl are required for platelet-activating factor-induced endothelial junctional disassembly and increase in vascular permeability. J Biol Chem 2009; 284: 5381-5394.

154. Chiba Y, Ishii Y, Kitamura S, et al. Activation of rho is involved in the mechanism of hydrogen-peroxide-induced lung edema in isolated perfused rabbit lung. Microvasc Res 2001; 62: 164-171.

155. Essler M, Retzer M, Bauer M, et al. Mildly oxidized low density lipoprotein induces contraction of human endothelial cells through activation of Rho/Rho kinase and inhibition of myosin light chain phosphatase. J Biol Chem 1999; 274 : 30361-30364.

156. Popoff MR, Geny B. Multifaceted role of Rho, Rac, Cdc42 and Ras in intercellular junctions, lessons from toxins. Biochim Biophys Acta 2009; 1788: 797-812.

157. Moolenaar WH, van Meeteren LA, Giepmans BN. The ins and outs of lysophosphatidic acid signalling. Bioessays 2004; 26: 870-881.

158. Parikh SM, Mammoto T, Schultz A, et al. Excess circulating angiopoietin-2 may contribute to pulmonary vascular leak in sepsis in humans. PLoS Med 2006; 3: e46.

159. van der Heijden M., van Nieuw Amerongen GP, Chedamni S, et al. The angiopoietin-Tie2 system as a therapeutic target in sepsis and acute lung injury. Expert Opin Ther Targets 2009; 13: 39-53.

160. Iwanicki MP, Vomastek T, Tilghman RW, et al. FAK, PDZ-RhoGEF and ROCKII cooperate to regulate adhesion movement and trailing-edge retraction in fibroblasts. J Cell Sci 2008; 121: 895-905.

161. Rumenapp U, Blomquist A, Schworer G, et al. Rho-specific binding and guanine nucleotide exchange catalysis by KIAA0380, a dbl family member. FEBS Lett 1999; 459: 313-318.
162. Murray D, Horgan G, Macmathuna P, et al. NET1-mediated RhoA activation facilitates lysophosphatidic acid-induced cell migration and invasion in gastric cancer. Br J Cancer 2008; 99: 1322-1329.

163. Kitzing TM, Sahadevan AS, Brandt DT, et al. Positive feedback between Dial, LARG, and RhoA regulates cell morphology and invasion. Genes Dev 2007; 21: 1478-1483.

164. Gebbink MF, Kranenburg O, Poland M, et al. Identification of a novel, putative Rho-specific GDP/GTP exchange factor and a RhoA-binding protein: control of neuronal morphology. J Cell Biol 1997; 137: 1603-1613.

165. Koga Y, Ikebe M. p116Rip decreases myosin II phosphorylation by activating myosin light chain phosphatase and by inactivating RhoA. J Biol Chem 2005; 280: 4983-4991.

166. Kakiashvili E, Speight P, Waheed F, et al. GEF-H1 mediates tumor necrosis factoralpha -induced Rho activation and myosin phosphorylation: Role in the regulation of tubular paracellular permeability. J Biol Chem 2009; 284: 11454-11466..

167. Papaharalambus C, Sajjad W, Syed A, et al. Tumor necrosis factor alpha stimulation of Racl activity. Role of isoprenylcysteine carboxylmethyltransferase. J Biol Chem 2005; 280: 18790-18796.

168. Williams LM, Lali F, Willetts K, et al. Rac mediates TNF-induced cytokine production via modulation of NF-kappaB. Mol Immunol 2008; 45: 2446-2454.

169. Haubert D, Gharib N, Rivero F, et al. PtdIns(4,5)P-restricted plasma membrane localization of FAN is involved in TNF-induced actin reorganization. EMBO J 2007; 26: 3308-3321.

170. Van Buul JD, Hordijk PL. Endothelial adapter proteins in leukocyte transmigration. Thromb Haemost 2009; 101: 649-655. 